\title{
Perturbed Marked Reduced forms of invariant subspaces *
}

\author{
Albert Compta, Josep Ferrer and Marta Peña \\ e-mails: albert.compta@upc.es, josep.ferrer@upc.es, marta.penya@upc.es
}

May 23, 2018

\begin{abstract}
The classification of invariant subspaces is an open problem related to other important ones like the Carlson problem. Here we obtain a reduced form of these invariant subspaces as a new tool to tackle these problems. In particular, it allows us to prove quite easily partial results already known. The key point is assigning to each invariant subspace a marked one (its marked type) in order to partition the set of invariant subspaces in a finite number of subsets (the marked classes), each one containing only one marked subspace. Next, we parametrize (minimally) each marked class by means of the so-called PM reduced families, so that representatives of an invariant subspace (its PM reduced forms) appear in just one of these families.
\end{abstract}

AMS Mathematics Subject Classification : 15A21, 93B10, 15A03.

Keywords: endomorphism, invariant subspaces, marked subspaces.

\section{Introduction}

Given an $N$-dimensional $\mathbb{C}$-vector space $E$, we consider the study of pairs $(\mathcal{V}, f)$, where $f$ runs over $\operatorname{End}(E)$ and, for each one, $\mathcal{V}$ runs over the $f$-invariant subspaces. We recall that for an endomorphism $f \in E n d(E)$, a subspace $\mathcal{V} \subset E$ is called $f$-invariant if $f(\mathcal{V}) \subset \mathcal{V}$, and two invariant pairs $(\mathcal{V}, f)$ and $\left(\mathcal{V}^{\prime}, f^{\prime}\right)$ are called equivalent if there is an automorphism $\varphi$ such that $\varphi(\mathcal{V})=\mathcal{V}^{\prime}$ and $\varphi \circ f=f^{\prime} \circ \varphi$. The classification of invariant pairs according to this equivalence relation is an open problem, with partial results for quite particular cases: [11] and [4] for monogenic subspaces; in [12] one proves that there is a finite number of equivalence classes if the degree of the minimal polynomial prime factors is less than or equal to 5 , and one asserts that the classification problem is "wild" when this degree is greater than 6 .

We can restrict ourselves (see section 2.1) to $f$ being a nilpotent endomorphism with a prefixed Segre characteristic $p=\left(p_{1}, \ldots, p_{n}\right), \mathcal{V}$ being a $d$-dimensional $f$-invariant subspace and the restriction of $f$ to $\mathcal{V}$ having a prefixed Segre characteristic $q=\left(q_{1}, \ldots, q_{m}\right)$. We denote by $\operatorname{Inv}(p, q)$

\footnotetext{
*Supported by the project MTM2017-90682-REDT of ALAMA net.
} 
the set of these invariant pairs (in [13] one shows that it is a differentiable manifold). Moreover, if two invariant pairs are equivalent then also the quotient endomorphisms must have the same Segre characteristic, but there are no explicit criteria in order to determine if a quotient Segre characteristic is compatible with $p$ and $q$ (the Carlson problem: see [2], [3]).

Our basic idea is exploiting the special structure and the nice properties of the marked subspaces, that is, the invariant subspaces having a Jordan basis of the restriction which can be extended to a Jordan basis of $f$ (see sections 2.3 and 7.3). The conclusion is that the study of the invariant subspaces in $\operatorname{Inv}(p, q)$ can be reduced to study a finite number of parametrised families in it (the PM reduced families), each one containing just a marked subspace.

In order to do that, our first goal is to assign to each invariant subspace in $\operatorname{Inv}(p, q)$, a marked subspace which we call its marked type (definition 4.4). Hence, the set $\operatorname{Inv}(p, q)$ is partitioned into a finite number of so-called marked classes, each one formed by the invariant subspaces having the same marked type. By means of this new classification item, the study of invariant subspaces (in particular the Carlson problem, the classification problem,...) can be reduced to each marked class in $\operatorname{Inv}(p, q)$.

Then, our second goal is the obtention of a (minimally) parametrised family of each marked class, where representatives of all the invariant subspaces having the same marked type (and only of them) appear. Each one (non unique, in general) is called a PM reduced form of the corresponding invariant subspace. Indeed, we obtain (see theorem 5.5) a quite simple parametrised family (the PM reduced family), centered at the marked subspace. Therefore, for example, the Carlson problem is reduced to discuss the possible quotient Segre characteristics according to the values of these parameters. In particular, concerning the classification problem, one derives easily all the partial results known till now (see section 6).

We point out that we use the vectorial representation of invariant subspaces (see section 2.2): the coordinates of the vectors of a Jordan basis of the restriction to the subspace in a Jordan basis of the space. In addition, the constructions using vectorial representation are mainly reduced to pivot transformations, adapted to the special pattern of the matrices representing invariant subspaces: see corollary 4.5 for the marked type, and definition 5.4 for the PM reduced forms.

Finally, we remark that a geometrical approach is included for interested people (section 7), although the paper can be understood without this last section. In particular, we see (corollary 7.15) that the marked type of a non-marked class is the greatest marked class which intersects its boundary. That is to say, among the marked subspaces such that the considered invariant subspace can be obtained as a perturbation of them, we select the one whose equivalence class has the maximal dimension. Moreover, we see (corollary 7.7) that the PM parametrised families are subfamilies of an Arnold miniversal deformation of the corresponding marked subspace, where the invariant subspace having other marked type has been eliminated.

The paper is organized as follows. In section 2 we summarize the previous results and notation needed for the development of our work. Specifically, we review the invariant subspaces and their vectorial representation. We continue with the marked subspaces and recall the vectorial representations relating them.

In section 3 we study the pivot transformations allowed in the vectorial representation.

In section 4 we introduce the "marked type" for any invariant subspace in $\operatorname{Inv}(p, q)$ or equivalently the "marked indices" (definition 4.4) obtained by means of an algorithm (named M 
algorithm in definition 4.1) from any vectorial representation of the subspace, and characterized in theorem 4.3.

In the section 5 we define the perturbed marked $(\mathrm{PM})$ reduced families parameterizing each marked class and the PM reduced forms for any invariant subspace in it (definition 5.4) along with an algorithm for calculating a reduced form from any vectorial representation of it.

In section 6 we present an example of classification (including the solutions of the Carlson problem and the Littlewood Richardson sequences) and we apply our techniques to the two particular cases already known (see [4] and [12] respectively): monogenic invariant subspaces, endomorphisms whose minimal polynomial have degree lower than 6 , when there is a finite number of classes.

Finally, in section 7 we present a geometrical approach, relating our study with geometric structures and miniversal deformations.

In all the paper we denote by $M_{N}(\mathbb{C})$ the complex $N$-square matrices and $M_{N, d}(\mathbb{C})$ the ones having $N$ rows and $d$ columns: In all the cases, if $M$ is a subset of matrices, then $M^{*}$ means those having the maximal rank.

\section{Preliminaries}

\subsection{Invariant Subspaces}

We recall some definitions and results concerning invariant subspaces.

Definition 2.1 Let $f \in \operatorname{End}(E)$ be an endomorphism. A subspace $\mathcal{V} \subset E$ is called invariant (or $f$-invariant) if $f(\mathcal{V}) \subset \mathcal{V}$.

Then, we denote by $\hat{f}$ the restriction of $f$ to $\mathcal{V}$ and $\tilde{f}$ the quotient endomorphism of $E / \mathcal{V}$ induced by $f$.

We denote by $\operatorname{Inv}_{d}(E)$ the set of invariant pairs $(\mathcal{V}, f)$, where $f \in \operatorname{End}(E), \mathcal{V}$ is an $f$-invariant subspace and $\operatorname{dim}(\mathcal{V})=d$. For a fixed $f \in \operatorname{End}(E)$ we denote by $\operatorname{Inv}_{d}(f)$ the set of d-dimensional $f$-invariant subspaces of $E$, which we consider a subset of $\operatorname{Inv}_{d}(E)$ by means of the identification: $\operatorname{Inv}_{d}(f) \equiv \operatorname{Inv}_{d}(f) \times\{f\} \subset \operatorname{Inv}_{d}(E)$.

Then, if $A$ is the matrix of $f$ in some basis of $E$, we say that $\mathcal{V}$ is an $A$-invariant subspace, that $(\mathcal{V}, A)$ is an invariant pair and so on.

Definition 2.2 Two invariant pairs $(\mathcal{V}, f)$ and $\left(\mathcal{V}^{\prime}, f^{\prime}\right)$ are called equivalent if there is $\varphi \in$ Aut $(E)$ such that $\varphi(\mathcal{V})=\mathcal{V}^{\prime}$ and $\varphi \circ f=f^{\prime} \circ \varphi$. We denote by $\widetilde{(\mathcal{V}, f)}$ the equivalence class of $(\mathcal{V}, f)$.

If $A, A^{\prime}$ are the matrices of $f, f^{\prime}$ in respective bases of $E$, then, $(\mathcal{V}, A)$ and $\left(\mathcal{V}^{\prime}, A^{\prime}\right)$ are equivalent if there is a matrix of change of basis $S$ such that $\mathcal{V}^{\prime}=S^{-1} \mathcal{V}$ and $A^{\prime}=S^{-1} A S$.

Bearing in mind the decomposition $\mathcal{V}=\oplus_{\lambda}\left(\operatorname{Ker}(f-\lambda I)^{N} \cap \mathcal{V}\right)$, where $\lambda$ runs over the eigenvalues of $f$, from now on we restrict ourselves to $f$ being nilpotent. 
Definition 2.3 $A$ partition $\alpha=\left(\alpha_{1}, \alpha_{2}, \ldots, \alpha_{m}, 0, \ldots\right)$, will be any nonincreasing finite sequence of non negative integers

$$
\alpha_{1} \geq \alpha_{2} \geq \cdots \geq \alpha_{m}>0
$$

Its conjugate partition $\alpha^{*}$ is defined by $\alpha_{j}^{*}=\#\left\{1 \leq i \leq m: \alpha_{i} \geq j\right\}$.

Definition 2.4 If $f$ is a nilpotent endomorphism, the Weyr characteristic is $\alpha=\left(\operatorname{dim} K \operatorname{erf}, \operatorname{dim} K e r f^{2}-\right.$ $\left.\operatorname{dim} K \operatorname{erf}, \operatorname{dim} K e r f^{3}-\operatorname{dim} K e r f^{2}, \ldots\right)$ and its conjugate partition is the Segre characteristic, formed by the sizes of Jordan blocks.

When convenient, we focus on the indecomposable invariant pairs (or subspaces):

Definition 2.5 An invariant pair $(\mathcal{V}, f) \in \operatorname{Inv}_{d}(E)$ is called indecomposable if there are no two non-null invariant pairs $(\mathcal{U}, f)$ and $\left(\mathcal{U}^{\prime}, f\right)$ such that $\mathcal{U} \oplus \mathcal{U}^{\prime}=E$ and $\mathcal{V}=(\mathcal{V} \cap \mathcal{U}) \oplus\left(\mathcal{V} \cap \mathcal{U}^{\prime}\right)$.

Otherwise, $\mathcal{V} \cap \mathcal{U}$ (as well as $\mathcal{V} \cap \mathcal{U}^{\prime}$ ) is called an invariant component of $\mathcal{V}$.

The following classification items are trivial:

Lemma 2.6 Let $(\mathcal{V}, f)$ be an invariant pair being $f$ nilpotent. Then, for any other $\left(\mathcal{V}^{\prime}, f^{\prime}\right)$ equivalent to it we have:

(i) $f^{\prime}$ is also nilpotent, with the same Segre characteristic as $f$.

(ii) $\operatorname{dim} \mathcal{V}^{\prime}=\operatorname{dim} \mathcal{V}$ (and $\left(\mathcal{V}^{\prime}, f^{\prime}\right)$ is indecomposable if $(\mathcal{V}, f)$ is so).

(iii) The restrictions $\hat{f}$ and $\hat{f}^{\prime}$ have the same Segre characteristic.

(iv) The quotient endomorphisms $\tilde{f}$ and $\tilde{f}^{\prime}$ have the same Segre characteristic.

Therefore, in order to classify the invariant pairs $(\mathcal{V}, f)$, we can restrict ourselves to the following situation:

Definition 2.7 Let us consider two partitions of integers $p=\left(p_{1}, \ldots, p_{n}\right), q=\left(q_{1}, \ldots, q_{n}\right)$ such that $p_{1}+\cdots+p_{n}=N, q_{1}+\cdots+q_{n}=d, p_{1} \geq \ldots p_{n}>0, q_{1} \geq \ldots q_{m}>q_{m+1}=\cdots=q_{n}=0$ and $p_{i} \geq q_{i}$ for $i=1, \ldots, n$. Then, we say that $q$ is compatible with $p$.

(1) We denote by $\operatorname{Inv}(p, q)$ the set of invariant pairs $(\mathcal{V}, f) \in \operatorname{Inv}_{d}(E)$ verifying that $f$ is nilpotent having Segre characteristic $p$ and the restriction $\hat{f}$ having Segre characteristic $q$.

(2) We denote by $J_{p}$ (respectively $J_{q}$ ) the nilpotent lower Jordan matrix having Segre characteristic $p$ (respectively $q$ ).

(3) We denote by $\operatorname{Inv} v_{q}\left(J_{p}\right)$ the subset of $\operatorname{Inv} v_{d}\left(J_{p}\right)$ whose restriction has Segre characteristic $q$. Then, as in definition 2.1, we identify: $\operatorname{Inv}_{q}\left(J_{p}\right) \equiv \operatorname{Inv}_{q}\left(J_{p}\right) \times\left\{J_{p}\right\} \subset \operatorname{Inv}(p, q)$.

Remark 2.8 We do not prefix the Segre characteristic of the quotient endomorphism $\tilde{f}$ because one does not know a direct criterium to it being compatible with $p$ and $q$ (the Carlson problem). An implicit criterium is that these three Segre characteristics must be related by a so-called Littlewood Richardson sequence. When it happens, in [3] one constructs explicitly an invariant pair $(\mathcal{V}, f)$ having these desired Segre characteristics. See also [2]. 


\subsection{The vectorial representations in $B L D^{*}(p, q)$}

The following lemma summarizes the matricial representations of invariant pairs $(\mathcal{V}, f)$.

Lemma 2.9 Let $f, f^{\prime} \in \operatorname{End}(E)$ and $A, A^{\prime}$ be their matrices in respective bases. Let us consider a d-dimensional subspace $\mathcal{V} \subset E$.

(1) If (and only if) the columns of $S \in M_{N}^{*}(\mathbb{C})$ form a basis of $E$ adapted to $\mathcal{V}$ (that is, an extension of a basis of $\mathcal{V}$ ) one has $S^{-1} \mathcal{V}=\mathbb{C}^{d} \times\{0\} \equiv \mathbb{C}^{d}$

Then, $\mathcal{V}$ is $f$-invariant if and only if $S^{-1} A S$ has the form $A_{0}=\left(\begin{array}{cc}A_{1} & A_{3} \\ 0 & A_{2}\end{array}\right), \quad$ with $A_{1} \in$ $M_{d}(\mathbb{C})$. Moreover, $A_{1}$ is the matrix of the restriction $\hat{f}$ in the corresponding basis of $\mathcal{V}$ and $A_{2}$ is the matrix of the quotient-endomorphism $\hat{f}$ in the induced basis of $E / \mathcal{V}$.

(2) If $A_{0}, A_{0}^{\prime}$ are matricial representations of $(\mathcal{V}, A)$ and $\left(\mathcal{V}^{\prime}, A^{\prime}\right)$ as above, then these invariant pairs are equivalent if and only if $A_{0}, A_{0}^{\prime}$ are so. Moreover, if $A_{0}^{\prime}=T^{-1} A_{0} T$, then also $T$ has the form $\left(\begin{array}{cc}T_{1} & T_{3} \\ 0 & T_{2}\end{array}\right)$, and $\quad A_{1}^{\prime}=\left(T_{1}\right)^{-1} A_{1} T_{1}, \quad A_{2}^{\prime}=\left(T_{2}\right)^{-1} A_{2} T_{2}$.

However, it is not easy to achieve that a matrix as $A_{0}$ above has a prefixed Segre characteristic. In order to do that, we will mainly use the following vectorial representations of an invariant pair. We consider Jordan bases of $f$, so that $(\mathcal{V}, f) \in \operatorname{Inv}(p, q)$ is represented by pairs of the form $\left(S p(Y), J_{p}\right)$ where the columns of $Y \in M_{N, d}^{*}(\mathbb{C})$ are the coordinates of a basis of $\mathcal{V}$ in the considered Jordan basis of $E$. If in addition the columns of $Y$ form a Jordan basis of the restriction $\hat{f}$, then we say that $Y$ is a vectorial representation of $(\mathcal{V}, f)$. We will see the pattern of these vectorial representations.

Definition 2.10 Let $p, q$ be as in definition 2.7.

1. A matrix is called lower diagonal $(L D)$ if it is a lower triangular matrix constant along the diagonals.

2. A partitioned matrix whose blocks are LD matrices will be called block lower diagonal (BLD).

3. We denote by $B L D(p, q)$ the BLD matrices with respect to the block partition $(p, q)$, that is to say, its rows are partitioned according to $p=\left(p_{1}, p_{2}, \ldots, p_{i}, \ldots, p_{n}\right)$ and its columns are partitioned according to $q=\left(q_{1}, q_{2}, \ldots, q_{j}, \ldots, q_{m}\right)$.

4. If $X \in B L D(p, q)$, we denote by $X_{i j}$ the block in $M_{p_{i}, q_{j}}(\mathbb{C})$, by $D_{i j}^{k}$ the $k$-diagonal of this block, where the first diagonal is the element in the left bottom corner, and by $x_{i j}^{k}$ the entries in $D_{i j}^{k}$. Then $x_{i j}^{k}=0$ if $k>\min \left(p_{i}, q_{j}\right)$.

5. In each block $X_{i j}$, we say that the entry $x_{i j}^{k}$ or the diagonal $D_{i j}^{k}$ has $k$ height, $\left(p_{i}-k\right)$ depth and $\left(q_{j}-k\right)$ horizontal depth.

6. We refer to the entry $x_{i j}^{q_{j}}$ or the diagonal $D_{i j}^{q_{j}}$ as the main ones. In particular, $x_{i j}^{q_{j}}=0$ if $p_{i}<q_{j}$.

We refer to $X_{\text {main }}=\left(x_{i j}^{q_{j}}\right)_{1 \leq i \leq n, 1 \leq j \leq m}$, as the main submatrix. 
7. If $h_{i j}=\max \left\{k: x_{i j}^{k} \neq 0\right\}$, we refer to the entry $x_{i j}^{h_{i j}}$ or the diagonal $D_{i j}^{h_{i j}}$ as the maximal ones.

8. We define $I_{i j}^{k}=\left(\begin{array}{cc}0 & 0 \\ I_{k} & 0\end{array}\right) \in M_{p_{i}, q_{j}}(\mathbb{C}) \quad$ for $\quad 1 \leq k \leq \min \left(p_{i}, q_{j}\right)$, so that we have $X_{i j}=\sum_{1 \leq k \leq h_{i j}} x_{i j}^{k} I_{i j}^{k}$. In particular, if $X_{i j}=I_{i j}^{k}$ we say that $X_{i j}$ is an unitary block, and the main unitary one if $k=q_{j}$.

9. We define a block row and a block column as $X_{i *}=\left(X_{i 1}, X_{i 2}, \ldots, X_{i m}\right)$ and $X_{* j}=$ $\left(X_{1 j}^{t}, X_{2 j}^{t}, \ldots, X_{n j}^{t}\right)^{t}$, respectively.

10. We denote as parameters the non-zero variable entries.

Example 2.11 $X \in B L D((3,2,1),(2,1))$ and its main submatrix $X_{\text {main }}$ are of the form

$$
X=\left[\begin{array}{cc|c}
0 & 0 & 0 \\
x_{11}^{2} & 0 & 0 \\
x_{11}^{1} & x_{11}^{2} & x_{12}^{1} \\
\hline x_{21}^{2} & 0 & 0 \\
x_{21}^{1} & x_{21}^{2} & x_{22}^{1} \\
\hline x_{31}^{1} & 0 & x_{32}^{1}
\end{array}\right], \quad X_{\text {main }}=\left[\begin{array}{cc}
x_{11}^{2} & x_{12}^{1} \\
x_{21}^{2} & x_{22}^{1} \\
0 & x_{32}^{1}
\end{array}\right]
$$

It is well known that:

Lemma 2.12 In the above notation:

1. $X \in B L D(p, q)$ has maximal rank if and only if $\operatorname{rank}\left(X_{\text {main }}\right)=m$.

2. $B L D^{*}(p, p)$ is the closed subgroup of $G l(N)$ formed by the non singular matrices which commute with $J_{p}$ (the lower Jordan nilpotent matrix having Segre characteristic $p$ ). Thus, $P \in B L D^{*}(p, p)$ is a change of Jordan bases of $J_{p}$.

3. Analogously for $B L D^{*}(q, q) \subset G l(d)$. Thus, $Q \in B L D^{*}(q, q)$ is a change of Jordan bases of $J_{q}$.

As announced, by means of a Jordan basis, any invariant pair $(\mathcal{V}, f) \in \operatorname{Inv}(p, q)$ can be represented by one of the form $\left(S p(Y), J_{p}\right)$ where $Y \in B L D^{*}(p, q)$ :

Proposition 2.13 [8] Let $(p, q)$ and $J_{p}$ be as in definition 2.7.

1. If $Y \in B L D^{*}(p, q)$, then $\left(S p(Y), J_{p}\right) \in \operatorname{Inv}(p, q)$ and the columns of $Y$ form a Jordan basis of $S p(Y)$.

2. Conversely, for any $(\mathcal{V}, A) \in \operatorname{Inv}(p, q)$ and any Jordan basis $S$ of $A$ we have $S^{-1} A S=J_{p}$ and $S^{-1} \mathcal{V}=S p(Y), \quad$ for some $Y \in B L D^{*}(p, q)$.

3. $B L D^{*}(q, q)$ acts freely on $B L D^{*}(p, q)$ by right multiplication and for any $Y, Y^{\prime} \in B L D^{*}(p, q)$ : $S p(Y)=S p\left(Y^{\prime}\right) \Leftrightarrow Y^{\prime}=Y Q$ for some $Q \in B L D^{*}(q, q)$. 
4. $B L D^{*}(p, p)$ acts freely on $B L D^{*}(p, q)$ by left multiplication and for any $Y, Y^{\prime} \in B L D^{*}(p, q)$ : $\left(S p(Y), J_{p}\right) \sim\left(S p\left(Y^{\prime}\right), J_{p}\right) \Leftrightarrow Y^{\prime}=P Y Q$ for some $P \in B L D^{*}(p, p)$ and $Q \in B L D^{*}(q, q)$.

Definition 2.14 We say that $Y \in B L D^{*}(p, q)$ is a vectorial representation of $(\mathcal{V}, A)$ or of its class $\widetilde{(\mathcal{V}, A)}$ if the two pairs are equivalent. We also say that $Y \in B L D^{*}(p, q)$ is a vectorial representation of $\mathcal{V}$ if one assumes $A=J_{p}$. When (4) in proposition 2.13 holds, we simply say that $Y, Y^{\prime} \in B L D^{*}(p, q)$ are equivalent, so that $\tilde{Y}=\left\{P Y Q: p \in B L D^{*}(p, p), Q \in B L D^{*}(q, q)\right\}$.

Therefore, the classification of invariant pairs in $\operatorname{Inv}(p, q)$ is equivalent to the classification of $Y \in B L D^{*}(p, q)$ with regard to the above equivalence relation. As a direct application, we have:

Proposition 2.15 Let $Y \in B L D^{*}(p, q)$ be a vectorial representation of $\left(\mathcal{V}, J_{p}\right) \in \operatorname{Inv}(p, q)$.

(1) Let us assume that for a certain $1 \leq j \leq m$ we have that for each $1 \leq i \leq n$, if $Y_{i j} \neq 0$, then $Y_{i s}=0$ for all $s \neq j$. Then, the columns of the block $Y_{* j}$ span a monogenic invariant component of $\mathcal{V}$.

(2) In addition, let us assume that for a certain set of column indices $L=\left\{j_{1}, \ldots, j_{k}\right\}$ we have that for any $s \notin L Y_{i s}=0$ if $Y_{i j_{t}} \neq 0$ for some $1 \leq t \leq k$. Then, $S p\left(Y_{* j_{1}}, \ldots, Y_{* j_{k}}\right)$ is an invariant component of $\mathcal{V}$ (which is monogenic if $k=1$ as in (1)).

\subsection{Marked subspaces}

The marked subspaces will play a key role in our approach. See [9] for a full geometric characterization and classification. Here we recall the basic definitions and properties which we will need in the sequel.

Definition 2.16 Let $f \in \operatorname{End}(E)$. An invariant subspace $\mathcal{V} \subset E$ is called $f$-marked if there is some Jordan basis of $\mathcal{V}$ which can be extended to a Jordan basis of $E$.

Example 2.17 Let $f \in \operatorname{End}(E)$ be nilpotent, with $p=(3,1)$. Let us consider a Jordan basis $e_{1}, f\left(e_{1}\right), f^{2}\left(e_{1}\right), e_{2}$. For $q=(2,0)$, the subspace $\mathcal{V}_{1}=S p\left(f\left(e_{1}\right), f^{2}\left(e_{1}\right)\right) \in \operatorname{Inv}(p, q)$ is marked, but $\mathcal{V}_{2}=S p\left(f\left(e_{1}\right)+e_{2}, f^{2}\left(e_{1}\right)\right) \in \operatorname{Inv}(p, q)$ is not.

The following lemma collects some trivial properties of marked subspaces:

Lemma 2.18 Let $f \in \operatorname{End}(E)$

(1) If a vectorial subspace is equivalent to a $f$-marked one, then it is also $f$-marked.

(2) In Inv $(p, q)$ the equivalence classes of marked subspaces are characterized by assigning injectively to each $q_{j}$-chain, $1 \leq j \leq m$, a $p_{i_{j}}$-chain, $1 \leq i_{j} \leq n$, compatible with it (that is, $\left.q_{j} \leq p_{i_{j}}\right)$. In other words, any class of marked subspaces is characterized by a permutation $p^{\prime}=\left(p_{i_{1}}, \ldots, p_{i_{m}}, \ldots, p_{i_{n}}\right)$ of $p$ with $q_{j} \leq p_{i_{j}}, 1 \leq j \leq m$ and $p_{i_{m+1}} \geq \ldots \geq p_{i_{n}}$. 
We select the following natural vectorial representation by means of a Jordan basis of the marked subspace extended to a Jordan basis of the whole space (not well-ordered).

Definition 2.19 In Inv $(p, q)$, let us consider a marked subspace characterized by the permutation $p^{\prime}=\left(p_{i_{1}}, \ldots, p_{i_{n}}\right)$ of $p$, as in the above lemma. Its canonical vectorial representation is $Y \in B L D^{*}(p, q)$ where all the entries are 0 except those in the main diagonals of $Y_{i_{1}, 1}, \ldots, Y_{i_{m}, m}$ which are 1 . We denote it by $Y\left(p^{\prime}\right)$.

Example 2.20 In $\operatorname{Inv}(p, q)$ with $p=(6,4,3,2)$ and $q=(4,2,1)$ :
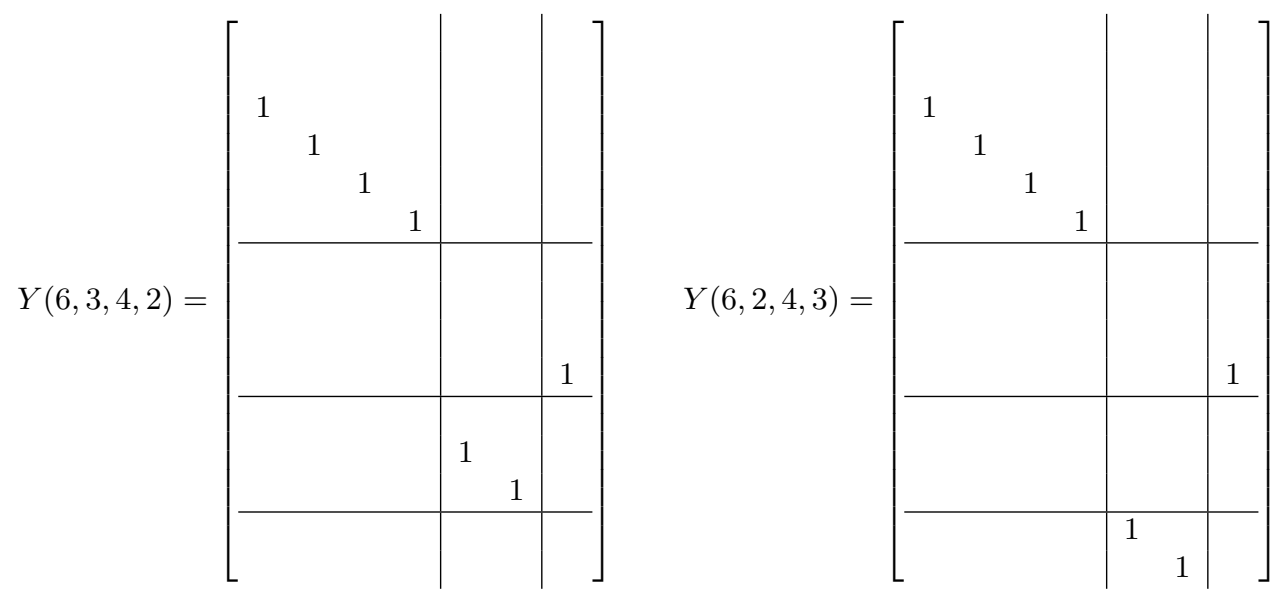

\section{BLD transformations}

We have seen (proposition 2.13) that the equivalence class of $Y \in B L D^{*}(p, q)$ is preserved by right (respectively left) multiplication by matrices in $B L D^{*}(q, q)$ (respectively $B L D^{*}(p, p)$ ). We will simplify $Y$ by means of these multiplications. Indeed they are equivalent to certain elementary transformations, as we precise in the following lemma:

Lemma 3.1 Let $Y \in B L D^{*}(p, q)$

(1) The right multiplication by matrices $Q \in B L D^{*}(q, q)$ is equivalent to a sequence of the following transformations, for each block-column $Y_{* j}$ :

(1.1) Multiplying $Y_{* j}$ by a non-zero scalar $\alpha$.

(1.2) Adding the last $t$ columns of $\alpha Y_{* j}$ (where $\alpha$ is an arbitrary scalar) to the first $t$ columns of any $Y_{* j^{\prime}}\left(\right.$ for any $1 \leq j^{\prime} \leq m$ and where $\left.t \leq \min \left(q_{j}, q_{j^{\prime}}\right)\right)$.

(2) The left multiplication by matrices $P \in B L D^{*}(p, p)$ is equivalent to a sequence of the following transformations, for each block-row $Y_{i *}$ :

(2.1) Multiplying $Y_{i *}$ by a non-zero scalar $\beta$.

(2.2) Adding the first $t$ rows of $\beta Y_{i *}$ (where $\beta$ is an arbitrary scalar) to the last $t$ rows of any $Y_{i^{\prime} *}\left(\right.$ for any $1 \leq i^{\prime} \leq n$ and where $\left.1 \leq t \leq \min \left(p_{i}, p_{i^{\prime}}\right)\right)$. 
Proof. Clearly (1.1) is equivalent to the right multiplication by $Q^{\prime}=\operatorname{diag}\left(I_{q_{1}}, \ldots, \alpha I_{q_{j}}, I_{q_{j}+1}, \ldots, I_{q_{m}}\right)$.

Concerning (1.2), let us write $v_{j}, f\left(v_{j}\right), \ldots, f^{q_{j}-1}\left(v_{j}\right)$, for $1 \leq j \leq m$, the columns of $Y$, which form a Jordan basis of $\mathcal{V}=S p(Y)$. Then (1.2) is equivalent to change $v_{j^{\prime}}, \ldots, f^{t}\left(v_{j^{\prime}}\right)$ by

$v_{j^{\prime}}+\alpha f^{q_{j}-t}\left(v_{j}\right), f\left(v_{j^{\prime}}+\alpha f^{q_{j}-t+1}\left(v_{j}\right), \ldots, f^{t}\left(v_{j^{\prime}}\right)+\alpha f^{q_{j}-1}\left(v_{j}\right)\right.$, resulting also a Jordan basis because $f\left(f^{t}\left(v_{j^{\prime}}\right)+\alpha f^{q_{j}-1}\left(v_{j}\right)\right)=f^{t+1}\left(v_{j^{\prime}}\right)$.

Clearly it is equivalent to the right multiplication by $I+Q^{\prime \prime} \in B L D^{*}(q, q)$ where the only non-zero block of $Q^{\prime \prime}$ is $Q_{j j^{\prime}}^{\prime \prime}=I_{j j^{\prime}}^{t}$.

Finally, it is clear that any matrix $Q \in B L D^{*}(q, q)$ is the product of matrices of the kind $Q^{\prime}$ and $I+Q^{\prime \prime}$ above.

Analogously for (2.1) and (2.2).

Definition 3.2 We refer to the transformations in (1) above as column BLD elementary transformations (column BLD-ETs). And to those in (2) as row BLD elementary transformations (row BLD-ETs).

Example 3.3 Let us consider $Y=\left[\begin{array}{ccc|cc}1 & 0 & 0 & 0 & 0 \\ 2 & 1 & 0 & 6 & 0 \\ 3 & 2 & 1 & 7 & 6 \\ \hline 4 & 0 & 0 & 8 & 0 \\ 5 & 4 & 0 & 9 & 8\end{array}\right]$

Applying (1.2) with: $t=1, \alpha=-\frac{1}{2}, j=2, j^{\prime}=1$ we obtain $Y Q=\left[\begin{array}{lll|ll}1 & 0 & 0 & 0 & 0 \\ 2 & 1 & 0 & 6 & 0 \\ 0 & 2 & 1 & 7 & 6 \\ \hline 4 & 0 & 0 & 8 & 0 \\ 1 & 4 & 0 & 9 & 8\end{array}\right]$.

Applying (2.2) with: $t=2, \beta=-4, i=1, i^{\prime}=2$ we obtain $P Y=\left[\begin{array}{ccc|cc}1 & 0 & 0 & 0 & 0 \\ 2 & 1 & 0 & 6 & 0 \\ 3 & 2 & 1 & 7 & 6 \\ \hline 0 & 0 & 0 & 8 & 0 \\ -3 & 0 & 0 & -13 & 8\end{array}\right]$.

As announced, we will use BLD-ETs in order to simplify the matrices $Y \in B L D^{*}(p, q)$. We will do this in the following way:

Proposition 3.4 Let $Y \in B L D^{*}(p, q)$

(1) Any block $Y_{i j}$ can be reduced, by means of column or row BLD-ETs, to an unitary one $I_{i j}^{h_{i j}}$ (see definition 2.10). In particular, it can be reduced to $I_{i j}^{q_{j}}$ when $Y_{i j}$ has non-zero main diagonal (only possible if $p_{i} \geq q_{j}$ ).

(1') In addition, let $Y_{i_{1} j_{1}}, \ldots, Y_{i_{s} j_{s}}$ be blocks of $Y$ placed in different block-columns (respectively block-rows), that is, $j_{1}, \ldots, j_{s}$ are different (respectively $i_{1}, \ldots, i_{s}$ are different). Then, by means of column BLD-ETs (respectively row BLD-ETs) all of them can be reduced to unitary blocks as in (1).

(2) Let $Y_{i j}=I_{i j}^{h_{i j}}$ be an unitary block: 
(2.1) By means of column BLD-ETs one can make 0 all the other diagonals in $Y_{i *}$ having the same or less height than $h_{i j}$, and the same or greater horizontal depth than $q_{j}-h_{i j}$ :

$$
\bar{y}_{i s}^{k}=0, \quad \text { if } \quad k \leq h_{i j} \quad \text { and } \quad q_{s}-k \geq q_{j}-h_{i j}
$$

(2.2) By means of row BLD-ETs one can make 0 all the other diagonals in $Y_{* j}$ having the same or less height than $h_{i j}$, and the same or greater depth than $p_{i}-h_{i j}$ :

$$
\bar{y}_{s j}^{k}=0, \quad \text { if } \quad k \leq h_{i j} \quad \text { and } \quad p_{s}-k \geq p_{i}-h_{i j}
$$

(2') In particular, if $Y_{i j}=I_{i j}^{q_{j}}$ is the main unitary block, then:

$$
\bar{Y}_{i s}=0, \quad \text { if } s>j \text { and } \bar{Y}_{s j}=0, \text { if } s<i
$$

In addition, if $q_{j}=p_{i}$, then: $\bar{Y}_{i s}=0$, if $s \neq j$ and $\bar{Y}_{s j}=0$, if $s \neq i$

Definition 3.5 We define as the $(i, j)$-column BLD-ET the composition of column BLD-ETs in (1) and (2.1)of proposition 3.4. We define as the right (left) $(i, j)$-column BLD-ET the same composition as above but only reducing the blocks in $Y_{i s}$ with $s \geq j(s \leq j)$. In the same way we define the $(i, j)$-row BLD-ET and the upper (lower) $(i, j)$-row BLD-ET.

Remark 3.6 We point out that when applying (2.1) also $y_{i^{\prime} s}^{h}$ could change. And analogously when applying (2.2). See the following example.

Example 3.7 Let us consider $Y$ below. By means of column BLD-ETs as in (1) above, we can obtain $Y_{1}=Y Q_{1}$. Next, by means of column BLD-ETs as in (2.1), we can obtain $Y_{2}=Y Q_{1} Q_{2}$. Finally, by means of row BLD-ETs as in (2.2), we can obtain $Y_{3}=P_{1} Y Q_{1} Q_{2}$.

$Y=\left[\begin{array}{ccc|cc|c}0 & 0 & 0 & 0 & 0 & 0 \\ y_{113} & 0 & 0 & 0 & 0 & 0 \\ y_{112} & \cdot & 0 & y_{122} & 0 & 0 \\ y_{111} & \cdot & \cdot & y_{121} & \cdot & y_{131} \\ \hline y_{213} & 0 & 0 & 0 & 0 & 0 \\ y_{212} & \cdot & 0 & 1 & 0 & 0 \\ y_{211} & \cdot & \cdot & y_{221} & 1 & y_{231} \\ \hline y_{312} & 0 & 0 & y_{322} & 0 & 0 \\ y_{311} & \cdot & 0 & y_{321} & \cdot & y_{331}\end{array}\right], Y_{1}=\left[\begin{array}{ccc|cc|c}0 & 0 & 0 & 0 & 0 & 0 \\ y_{113} & 0 & 0 & 0 & 0 & 0 \\ y_{112} & \cdot & 0 & y_{122} & 0 & 0 \\ y_{111} & \cdot & \cdot & \bar{y}_{121} & \cdot & y_{131} \\ \hline y_{213} & 0 & 0 & 0 & 0 & 0 \\ y_{212} & \cdot & 0 & 1 & 0 & 0 \\ y_{211} & \cdot & . & 0 & 1 & y_{231} \\ \hline y_{312} & 0 & 0 & y_{322} & 0 & 0 \\ y_{311} & \cdot & 0 & \bar{y}_{321} & \cdot & y_{331}\end{array}\right]$,

$Y_{2}=\left[\begin{array}{ccc|cc|c}0 & 0 & 0 & 0 & 0 & 0 \\ y_{113} & 0 & 0 & 0 & 0 & 0 \\ \bar{y}_{112} & \cdot & 0 & y_{122} & 0 & 0 \\ \bar{y}_{111} & \cdot & \cdot & \bar{y}_{121} & \cdot & \bar{y}_{131} \\ \hline y_{213} & 0 & 0 & 0 & 0 & 0 \\ 0 & \cdot & 0 & 1 & 0 & 0 \\ 0 & 0 & \cdot & 0 & 1 & 0 \\ \hline \bar{y}_{312} & 0 & 0 & y_{322} & 0 & 0 \\ \bar{y}_{311} & \cdot & 0 & \bar{y}_{321} & \cdot & \bar{y}_{331}\end{array}\right], Y_{3}=\left[\begin{array}{ccc|cc|c}0 & 0 & 0 & 0 & 0 & 0 \\ \bar{y}_{113} & 0 & 0 & 0 & 0 & 0 \\ \bar{y}_{112}^{\prime} & \cdot & 0 & 0 & 0 & 0 \\ \bar{y}_{111} & \cdot & \cdot & 0 & & \bar{y}_{131} \\ \hline y_{213} & 0 & 0 & 0 & 0 & 0 \\ 0 & \cdot & 0 & 1 & 0 & 0 \\ 0 & 0 & \cdot & 0 & 1 & 0 \\ \hline \bar{y}_{312}^{\prime} & 0 & 0 & y_{322} & 0 & 0 \\ \bar{y}_{311} & \cdot & 0 & 0 & \cdot & \bar{y}_{331}\end{array}\right]$

In addition we can reduce the block $Y_{11}$ to an unitary one by means of row BLD-ETs. It will be main if $\bar{y}_{113} \neq 0$.

On the other hand, it is not possible to obtain $y_{213}=0$ by means of BLD-ETs (although $\bar{y}_{113} \neq 0$, which has greater depth than $y_{213}$ ).

If $\bar{y}_{331} \neq 0$, we can make 0 the block $Y_{13}$ (by means of row BLD-ETs), but probably $Y_{12}$ will be changed. 
Remark 3.8 Although we will use only the above techniques, more general ones are possible. For example, if $D_{i j}^{h_{i j}}$ is a maximal diagonal (not necessarily the main one), then we can make 0 the diagonals $D_{i j^{\prime}}^{k}$ having $j^{\prime} \neq j, \quad k \leq h_{i j}$ or $j^{\prime}=j, \quad k<h_{i j}$.

We point out that if $D_{i^{\prime} j}^{k+\Delta} \neq 0$, then the diagonals $D_{i^{\prime} j^{\prime}}^{k+1}, \ldots, D_{i^{\prime} j^{\prime}}^{k+\Delta}$ are also changed.

\section{The marked type of an invariant subspace}

The key point of this paper is to partition $\operatorname{Inv}(p, q)$ in as many subsets as there are different classes of marked subspaces. The only marked class represented in each subset will be called the marked type of all the subspaces in it. In this section, for each invariant subspace we select its marked type. Hence, the study of the invariant subspaces in $\operatorname{In} v(p, q)$ is reduced to studying those having the same marked type.

In the next section, we will see that the invariant subspaces in each subset (and only them) are represented in a parametrised family centered in their marked type (the PM reduced family). The conclusion is that the set of vectorial representations of $\operatorname{Inv}(p, q)$ is partitioned in finitely many parametrised families (the PM reduced families) in such a way that each invariant subspace is represented in only the one centered in its marked type. Therefore, the study of invariant subspaces in $\operatorname{Inv}(p, q)$ can be reduced to each of these (finitely many) PM reduced families, each one centered in a marked subspace.

Firstly, the following lemma assigns indices $i_{1}, \ldots, i_{m}$, verifying (2) in lemma 2.18 , to each invariant subspace in $\operatorname{Inv}(p, q)$. Next theorem 4.3 shows that they are uniquely characterized. The conclusion is that they determine (definition 4.4) the so called marked type of the given invariant subspace.

Definition 4.1 We call the $\mathrm{M}$ algorithm the following one which assigns indices $1 \leq i_{1}, \ldots, i_{m} \leq$ $n$ to each $Y \in B L D^{*}(p, q)$.

For $j=1$, let $i_{1}$ be the greatest $1 \leq i \leq n$ such that the main diagonal of $Y_{i 1}$ is non-zero:

$$
D_{i_{1} 1}^{q_{1}} \neq 0, \quad D_{i 1}^{q_{1}}=0 \quad \text { if } \quad i>i_{1}
$$

Such $i_{1}$ index exists because $Y_{\text {main }}$ has full rank. Then, the main diagonals $D_{i_{1} j}^{q_{j}}$ for $j>1$ have equal or less height and equal horizontal depth than $D_{i_{1} 1}^{q_{1}}$, so they can become 0 by means of column BLD-ETs.

In the new equivalent matrix $Y^{1}, D_{i_{1} j}^{q_{j}}=0$ for $j>1$, so the only non-zero entry in the $i_{1}$ row of $Y_{\text {main }}^{1}$ is $y_{i_{1}, 1}$.

Next, for $j=2$, there is $i_{2} \neq i_{1}$ such that $D_{i_{2} 2}^{q_{2}} \neq 0, \quad D_{i 2}^{q_{2}}=0 \quad$ if $\quad i>i_{2}$ because $Y_{\text {main }}^{1}$ has full rank (and the second column cannot be 0). As above, by means of column $B L D$-ETs there is an equivalent matrix $Y^{2}$ with $D_{i_{2} j}^{q_{j}}=0$ for $j>2$.

And so on.

Example 4.2 Let us consider the parametrised family of subspaces represented by 


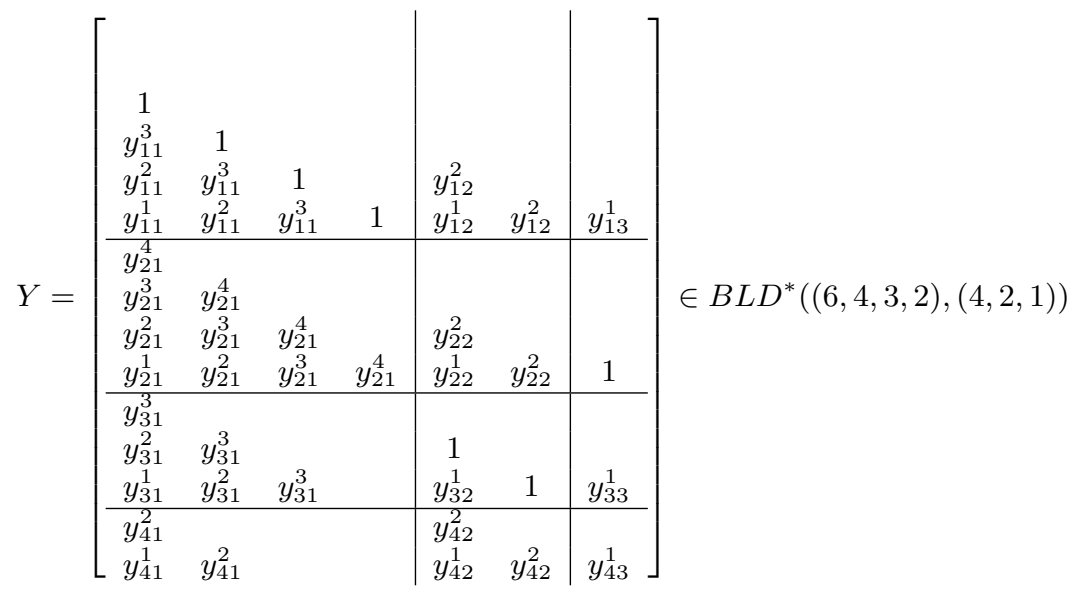

Each subspace in this family can be seen as a perturbation of several marked subspaces: $Y(6,3,4,2) ; Y(6,3,2,4)$, if $y_{43}^{1} \neq 0 ; Y(6,2,4,3)$, if $y_{42}^{2} \neq 0 ; Y(4,3,6,2)$, if $y_{31}^{1} \neq 0$; etc. But definition 4.1 selects one of them. Indeed, $i_{1}=2$ if $y_{21}^{4} \neq 0$, and $i_{1}=1$ otherwise.

In any case: $i_{2}=4$ if $y_{42}^{2} \neq 0$, and $i_{2}=3$ otherwise.

If $y_{21}^{4}=y_{42}^{2}=0$, then $i_{3}=4$ if $y_{43}^{1} \neq 0$, and $i_{3}=2$ otherwise.

Let us assume $y_{21}^{4}=0$ and $y_{42}^{2} \neq 0$, so that $i_{1}=1$ and $i_{2}=4$.

Then $i_{3}=3$ if $\bar{y}_{33}^{1}=y_{33}^{1}-y_{43}^{1} / y_{42}^{2} \neq 0$, and $i_{3}=2$ otherwise.

In the first case $i_{4}=2$ and in the second one $i_{4}=3$. The original parametrised family $Y$ has been reduced respectively to
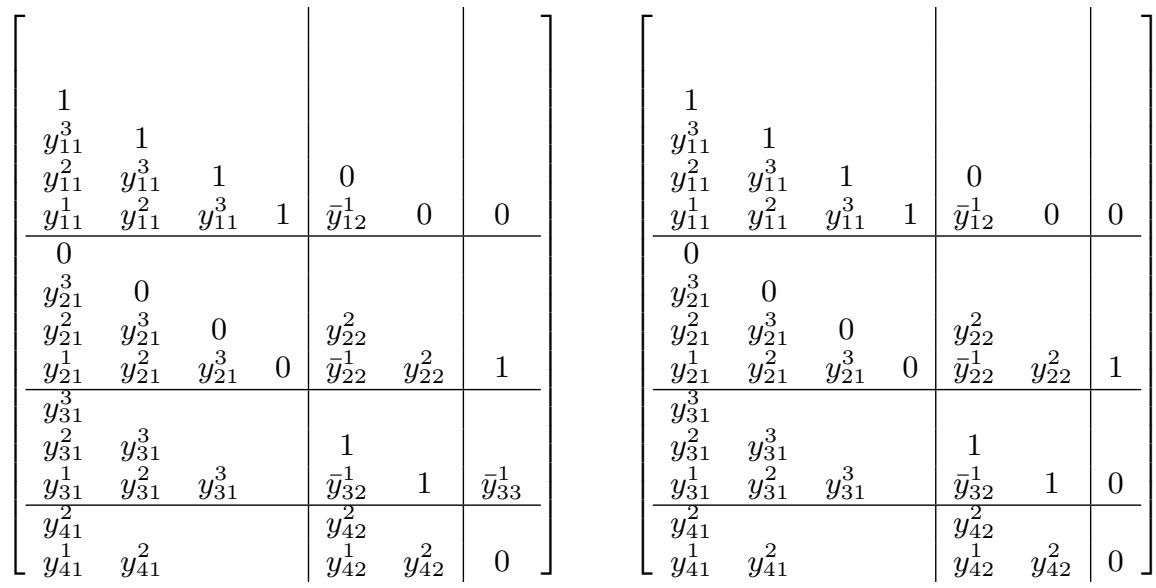

Theorem 4.3 Let $\mathcal{V} \in \operatorname{Inv}(p, q)$. There is a vectorial representation $Y \in B L D^{*}(p, q)$ such that the only non-zero main diagonals are $D_{i_{1} 1}^{q_{1}}, \ldots, D_{i_{m} m}^{q_{m}}$ for some different indices $1 \leq i_{1}, \ldots, i_{m} \leq$ $n$.

Conversely, if a vectorial representation has just one non-zero main diagonal in each column block and they are placed in different row blocks $1 \leq i_{1}^{\prime}, \ldots, i_{m}^{\prime} \leq n$, then $\left(p_{i_{1}^{\prime}}, \ldots, p_{i_{m}^{\prime}}\right)=$ $\left(p_{i_{1}}, \ldots, p_{i_{m}}\right)$ assuming that if $q_{j}=q_{j+s}, s>0$, then $i_{j} \geq i_{j+s}$. 
First of all we prove the existence:

Using the $\mathrm{M}$ algorithm we obtain a vectorial representation with non-zero main diagonals $D_{i_{1}, 1}^{q_{1}}, \ldots D_{i_{m}, m}^{q_{m}}$, placed in different block rows, such that $D_{i, j}^{q_{j}}=0$ if $i>i_{j}$.

Now, for $j=1$, by means of row BLD-ETs we make $D_{i, 1}^{q_{1}}=0$ if $i<i_{1}$ (possible because they have the same height and the same or greater depth), and the main diagonals in the other columns are not changed because $D_{i_{1}, j}=0$ for $j \neq 1$. Next, for $j=2$, again by means of row BLD-ETs we make $D_{i, 2}^{q_{2}}=0$ if $i<i_{2}$, and the main diagonals in the other columns are not changed because $D_{i_{2}, j}^{q_{j}}=0$ for $j \neq 2$ (indeed, by the M algorithm for $j>2$, and by the previous step for $j<2$ ).

And so on.

Conversely, the main diagonals $D_{i_{j} j}^{q_{j}}$ cannot be made 0 neither by row BLD-ETs (because the other non-null main diagonals in the column block $Y_{* j}$ are deeper) nor by column BLD-ETs (because the other non-null diagonals in the row block $Y_{i^{*}}$ have greater horizontal depth). Then, sizes of the blocks with non-zero main diagonal cannot be changed by BLD-ETs, and taking into account that if $q_{j}=q_{j+s}, s>0$, then $i_{j} \geq i_{j+s}$ (to avoid some irrelevant permutation of indices $i_{j}$ ), it results that $p_{i_{j}^{\prime}}=p_{i_{j}}$.

Definition 4.4 Let $\mathcal{V} \in \operatorname{Inv}(p, q)$ and $\left(i_{1}, \ldots, i_{m}\right)$ be the row indices obtained in theorem 4.3 (or in the $M$ algorithm in definition 4.1) from any representative $Y \in B L D^{*}(p, q)$. We number the remaining indices $1 \leq i_{m+1}, \ldots, i_{n} \leq n$ in increasing order. Then, we define the indices $p^{\prime}=\left(p_{i_{1}}, \ldots, p_{i_{n}}\right)$ as the marked indices of $\mathcal{V} \in \operatorname{Inv}(p, q)$ and the marked subspace represented by $Y\left(p^{\prime}\right)$ its marked type. The subset of $\operatorname{Inv}(p, q)$ formed by the invariant subspaces having marked indices $p^{\prime}$ will be called the marked class $p^{\prime}$.

Remark 4.5 The $M$ algorithm in definition 4.1 can be applied analogously to the main submatrix of any vectorial representation in order to obtain the marked type of an invariant subspace $\mathcal{V} \in \operatorname{Inv}(p, q)$. Let $Y \in B L D^{*}(p, q)$ be any vectorial representation of $\mathcal{V}$ and $Y_{\text {main }}=\left(y_{i j}^{q_{j}}\right) \equiv$ $\left(y_{i j}\right)$ be its main submatrix.

Let $i_{1}$ be such that $y_{i_{1} 1} \neq 0, y_{i 1}=0$ if $i>i_{1}$.

By means of right column ET, let us make $y_{i_{1} j}=0 \quad$ if $\quad j>1$.

Next, in the new main submatrix, let $i_{2}$ be such that $y_{i_{2} 2} \neq 0, y_{i 2}=0$ if $i>i_{2}$.

By means of right column ET, let us make $y_{i_{2} j}=0 \quad$ if $\quad j>2$.

And so on.

Example 4.6 In the above example, we have

$$
Y_{\text {main }}=\left(\begin{array}{ccc}
1 & y_{12}^{2} & y_{13}^{1} \\
y_{21}^{4} & y_{22}^{2} & 1 \\
0 & 1 & y_{33}^{1} \\
0 & y_{42}^{2} & y_{43}^{1}
\end{array}\right) \equiv\left(\begin{array}{ccc}
1 & y_{12} & y_{13} \\
y_{21} & y_{22} & 1 \\
0 & 1 & y_{33} \\
0 & y_{42} & y_{43}
\end{array}\right)
$$

If $y_{21}=0$, then $i_{1}=1$ and $Y_{\text {main }}$ becomes $Y_{\text {main }}^{\prime}$. If in addition $y_{42} \neq 0$, then $i_{2}=4$ and $Y_{\text {main }}$ is reduced to $Y_{\text {main }}^{\prime \prime}$, 


$$
Y_{\text {main }}^{\prime}=\left(\begin{array}{ccc}
1 & 0 & 0 \\
0 & y_{22} & 1 \\
0 & 1 & y_{33} \\
0 & y_{42} & y_{43}
\end{array}\right) \quad Y_{\text {main }}^{\prime \prime}=\left(\begin{array}{ccc}
1 & 0 & 0 \\
0 & y_{22} & \bar{y}_{32} \\
0 & 1 & \bar{y}_{33} \\
0 & y_{42} & 0
\end{array}\right)
$$

where $\bar{y}_{33}=y_{33}-\left(y_{43} / y_{42}\right), \bar{y}_{32}=1-\left(y_{22} y_{43} / y_{42}\right)$.

Next, $i_{3}=3$ if $\bar{y}_{33} \neq 0$ or $i_{3}=2$ if $\bar{y}_{33}=0$.

Finally $i_{4}=2$ or $i_{4}=3$ respectively.

\section{The perturbed marked (PM) reduced forms of an invariant subspace}

Let us construct for each marked type $Y \equiv Y\left(p^{\prime}\right) \in B L D^{*}(p, q)$, a parametrised family $Y(*)$ in $B L D^{*}(p, q)$ centered at $Y$ (that is, $Y$ appears just when all the parameters are 0 ) which contains representations of all (and only of them) invariant subspaces having marked type $Y$. Any representative of such an invariant subspace will be called a perturbed marked (PM) vectorial reduced form of it. It is not unique in general, so that it is not a canonical form. However, we will try to minimize the number of parameters.

From theorem 4.3, it is clear that all the invariant subspaces having the same marked indices (and only them) appear in the following parametrised family:

Corollary 5.1 Let $Y \equiv Y\left(p^{\prime}\right)$ be the canonical vectorial representation of a marked subspace in Inv $(p, q)$. Let us consider the parametrised family $Y(*)$ in $B L D^{*}(p, q)$ with general parameters $y_{i j}^{k}$ except in the main diagonals:

$$
\begin{gathered}
y_{i_{1} 1}^{q_{1}}=\ldots=y_{i_{m} m}^{q_{m}}=1 \\
y_{i j}^{q_{j}}=0 \quad \text { if } \quad i \neq i_{j}
\end{gathered}
$$

Then, this family contains representations of all the invariant subspaces having marked type $Y$, and only of them. In particular, $Y$ is the only marked subspace represented in it and a representative appears when (and only when) all the parameters are 0.

Example 5.2 In the conditions of example 4.2 the parametrised families
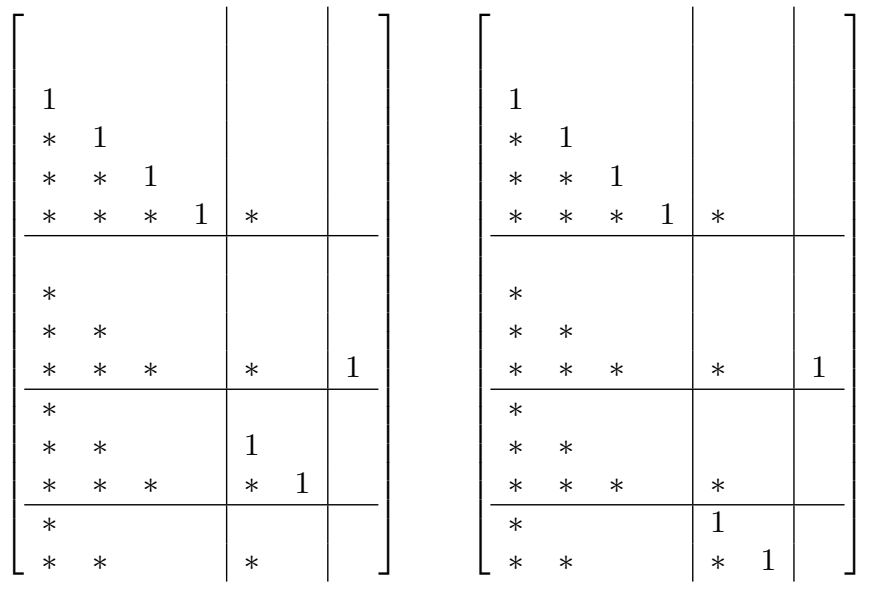
contain representations of all invariant subspaces having marked indices $(6,3,4)$ or $(6,2,4)$ respectively, and only of them.

We will simplify these parametrised families by improving the vectorial representations in theorem 4.3:

Theorem 5.3 Let $\mathcal{V} \in \operatorname{Inv}(p, q)$ have marked indices $p^{\prime}=\left(p_{i_{1}}, \ldots, p_{i_{n}}\right)$. There is a representative of it $Y \in B L D^{*}(p, q)$ such that the only non-zero main diagonals are $D_{i_{1} 1}^{q_{1}}, \ldots, D_{i_{m} m}^{q_{m}}$ and

(1) $Y_{i_{j}, j}=I_{i_{j}, j}^{q_{j}}, \quad Y_{i_{j}, s}=0$ if $s>j$,

(2) $Y_{i, j}=0$ if $i<i_{j}$,

(3) $y_{i_{j}, s}^{k}=0$ if $s<j, \quad k \leq q_{j}$,

(4) $y_{i, j}^{k}=0$ if $i>i_{j}, \quad k \leq p_{i}-p_{i_{j}}+q_{j}$.

Proof.

Using the $\mathrm{M}$ algorithm we obtain a vectorial representation $Y \in B L D^{*}(p, q)$ with non-zero main diagonals $D_{i_{1}, 1}^{q_{1}}, \ldots D_{i_{m}, m}^{q_{m}}$, placed in different block rows, such that $D_{i, j}^{q_{j}}=0$ if $i>i_{j}$.

We will obtain the desired representative in four steps. Adding one of the enumerated conditions in every step. In all of the BLD-ETs below we say increasing/decreasing order according to the order of the indices $i_{1}, \ldots, i_{m}$.

(1) By means of the right $\left(i_{j}, j\right)$-column BLD-ETs for $j=1, \ldots, m$ the block $Y_{i_{j}, j}$ becomes $I_{i_{j}, j}^{q_{j}}$ and the blocks $Y_{i_{j}, s}$, for $s>j$, become 0 because all their diagonals have the same or less height than $q_{j}$ and the same or greater horizontal depth than 0 (the height and depth of $D_{i_{j}, j}^{q_{j}}$ respectively). Notice that for $i_{j+k}>i_{j} D_{i_{j+k}, j}^{q_{j}}=0$, then the $\left(i_{j}, j\right)$-column BLD-ET does not change $D_{i_{j+k}, j+k}^{q_{j+k}} \neq 0$. Notice too that for $i_{j+k}<i_{j} D_{i_{j}, j+k}^{q_{j+k}}=0$ already, then the $\left(i_{j}, j\right)$-column BLD-ET does not change $D_{i_{j+k}, j+k}^{q_{j+k}} \neq 0$. We call $Y^{1}$ the new representative of $\mathcal{V}$.

(2) Now, by means of the upper $\left(i_{j}, j\right)$-row BLD-ETs applied in decreasing order, the blocks $Y_{i, j}^{1}$ with $i<i_{j}$ become 0 . Notice that we only transform the blocks $Y_{i_{s}, i_{j}}^{1}$ with $i_{s}<i_{j}, j<s \leq n$ because $Y_{i_{s}, i_{j}}^{1}=0$ for $i_{s}<i_{j}$ and $j>s$.

When any upper $\left(i_{j}, j\right)$-row BLD-ET is done, the new blocks $Y_{i, j}$ for $i<i_{j}$ obtained will not be changed by all the next upper $\left(i_{k}, k\right)$-row BLD-ETs because $i_{k}<i_{j}$ and the blocks $Y_{i_{k}, j}$ were already 0 . We call $Y^{2}$ the new representative of $\mathcal{V}$.

(3) We use the left $\left(i_{j}, j\right)$-column BLD-ETs in increasing order to reduce the blocks $Y_{i_{j}, k}^{2}$ for $k<j$. Notice that we only reduce the blocks $Y_{i_{j}, k}^{2}$, with $i_{k}<i_{j}$ because the others are already zero. Doing so in increasing order, the reduced blocks in the row $i_{j}$ will not be changed by the following transformations because $Y_{i_{j}, s}^{2}=0$ if $i_{s}>i_{j}$.

We call $Y^{3}$ the new representative of $\mathcal{V}$. Then, $y_{i_{j}, s}^{3, k}=0$ for $s<j$ and $k \leq q_{j}$. 
(4) Finally, again in decreasing order, we use the lower $\left(i_{j}, j\right)$-row BLD-ETs to reduce the blocks $Y_{i, j}^{3}$ for $i>i_{j}$. Notice that we only reduce the blocks $Y_{i_{k}, j}^{3}$, for $i_{k}>i_{j}$ and $k>j$, because $Y_{i_{k}, j}^{3}=0$ if $i_{k}>i_{j}$ and $k<j$. Once reduced $Y_{i_{k}, j}^{3}$, it will be not affected by another reduction because $Y_{i_{s}, j}^{3}=0$ for $i_{s}<i_{j}$.

With this operation all the diagonals of $Y_{i_{k}, j}^{3}$ for $i_{k}>i_{j}$ and $k>j$ with less height than $q_{j}$ and the same or greater depth than $p_{i_{j}}-q_{j}$ become 0 .

We call $Y^{4}$ the new representative of $\mathcal{V}$. Then, $y_{i, j, k}^{4}=0$ for $k \leq p_{i}-p_{i_{j}}+q_{j}$ if $i>i_{j}$.

Therefore we define:

Definition 5.4 (i) We call $\mathrm{PM}$ algorithm the simplification of $Y \in B L D^{*}(p, q)$ in theorem 5.3, that is to say:

(0) The $M$ algorithm.

(1) For $j=1, \ldots, m$, the right $\left(i_{j}, j\right)$-column BLD-ETs applied in the increasing order of the set of integers $\{1, \ldots, m\}$.

(2) For $j=1, \ldots, m$, the upper $\left(i_{j}, j\right)$-row BLD-ETs applied in the decreasing order of the set of integers $\left\{i_{1}, \ldots, i_{m}\right\}$.

(3) For $j=1, \ldots, m$, the left $\left(i_{j}, j\right)$-column BLD-ETs applied in the increasing order of the set of integers $\left\{i_{1}, \ldots, i_{m}\right\}$.

(4) For $j=1, \ldots, m$, the lower $\left(i_{j}, j\right)$-row BLD-ETs applied in the decreasing order of the set of integers $\left\{i_{1}, \ldots, i_{m}\right\}$.

(ii) Let $Y\left(p^{\prime}\right)$ be the canonical vectorial representation of a marked subspace in Inv $(p, q)$. The parametrised family $Y_{\text {red }}\left(p^{\prime}\right)(*) \in B L D^{*}(p, q)$ with parameters $y_{i j}^{h}$ satisfying the above conditions in theorem 5.3:

$$
\begin{gathered}
y_{i_{1} 1}^{q_{1}}=\ldots=y_{i_{m} m}^{q_{m}}=1 \\
y_{i_{s} j}^{k}=0 \quad \text { except } \quad i_{s}>i_{j}, \quad \max \left(q_{s}, p_{i_{s}}-p_{i_{j}}+q_{j}\right)<k<\min \left(q_{j}, p_{i_{s}}+1\right)
\end{gathered}
$$

where $q_{s}=0$ if $s>m$ and $p_{i_{m+1}} \geq \ldots \geq p_{i_{n}}$ will be called its perturbed marked (PM) reduced family.

(iii) For an invariant subspace having marked indices $p^{\prime}$, any representative in $Y_{\text {red }}\left(p^{\prime}\right)(*)$ (not unique, in general) is called a PM vectorial reduced form. In particular, the reduced forms obtained by means of the PM algorithm, starting from any vectorial representation.

Hence, we reformulate corollary 5.1:

Theorem 5.5 Let us consider a marked subspace in Inv $(p, q)$ and let $Y\left(p^{\prime}\right)$ be its canonical vectorial representation. Then, its PM reduced family $Y_{\text {red }}\left(p^{\prime}\right)(*)$ contains representatives (several, in general) of all invariant subspaces having marked indices $p^{\prime}$ and only of them. In particular, $Y\left(p^{\prime}\right)$ is the only marked subspace represented in it and its unique representative appears when all the parameters are 0 . 
Proof.

As we have proved in theorem 5.3, in a constructive way by means of an algorithm, every subspace in $\operatorname{Inv}(p, q)$ has a vectorial representation (not unique) in the only one family $Y_{\text {red }}\left(p^{\prime}\right)(*)$ where $p^{\prime}$ are its marked indices.

If we take a matrix in $Y_{\text {red }}\left(p^{\prime}\right)(*)$ with some non-zero parameter, it is impossible to eliminate it by BLD-ETs, so this matrix is not equivalent to $Y\left(p^{\prime}\right)$.

Example 5.6 In the above example 5.2 we obtain respectively
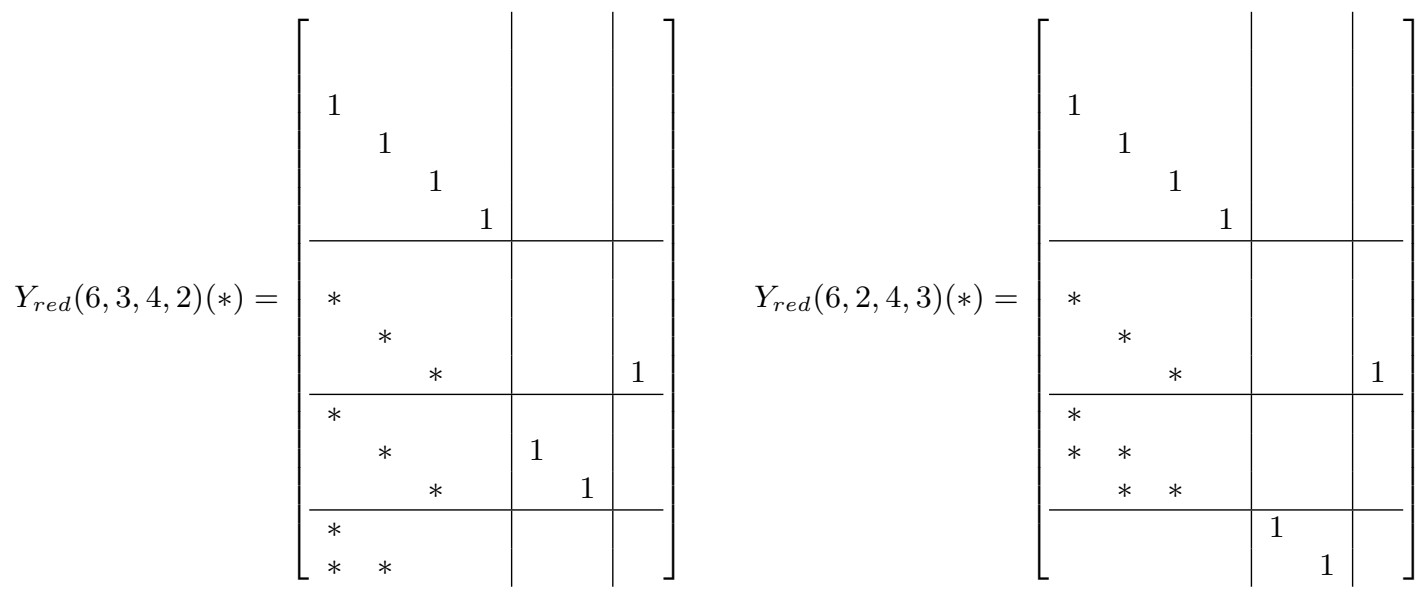

Notice that in $Y_{\text {red }}(6,2,4,3)(*)$ above the subspace spanned by the second column block is a monogenic invariant component (see proposition 2.15). In general, one has:

Corollary 5.7 Let $\mathcal{V} \in \operatorname{Inv}(p, q)$ have marked indices $p^{\prime}=\left(p_{i_{1}}, \ldots, p_{i_{m}}\right)$ and $Z(*) \equiv Y_{\text {red }}\left(p^{\prime}\right)(*)$ be the corresponding PM reduced family. Then,

(1) If $p_{i_{j}}=q_{j}$ for some $1 \leq j \leq m$, then $S p\left(Z_{* j}\right)$ is a (marked) monogenic invariant component of $\mathcal{V}$.

(2) If $q_{1}=\ldots=q_{m}$, then $S p\left(Z_{* m}\right)$ is a (marked) monogenic invariant component of $\mathcal{V}$.

(3) If $q_{1}=2$ and $q_{m}=1$, then $S p\left(Z_{* m}\right)$ is a (marked) monogenic invariant component of $\mathcal{V}$.

Remark 5.8 In order to improve the classification in Inv $(p, q)$, further classification items are suggested by the above result, obtaining more simplified reduced forms. For example, for each $m<s \leq n$ :

$$
k_{s}=\max \left\{k: y_{i_{s} j}^{k} \neq 0\right\} ; \quad j_{s}=\max \left\{j: y_{i_{s} j}^{k_{s}} \neq 0\right\}
$$

Then, the block $Y_{i_{s} j_{s}}$ can be reduced to the unitary one $I_{i_{s}, j_{s}, k_{s}}$.

For example, in $Y_{\text {red }}(6,2,4,3)(*)$ above:

$$
k_{s}=3, \quad 2 \quad \text { or } 0 ; \quad j_{s}=1 \text {. }
$$

In particular, if $k_{s}=0$ for some $m<s \leq n$, then the invariant subspace is decomposable. 


\section{Applications}

Theorem 5.3 solves the classification problem in $\operatorname{Inv}(p, q)$ for the most important already known particular cases. Previously we present an example of classification.

\subsection{A classification example}

Example 6.1 Let us consider $\operatorname{Inv}(p, q)$ with $p=(3,2,1), q=(2,1)$. The possible marked subspaces are $Y(3,2,1), Y(3,1,2), Y(2,3,1)$ and $Y(2,1,3)$, and the corresponding PM reduced families:
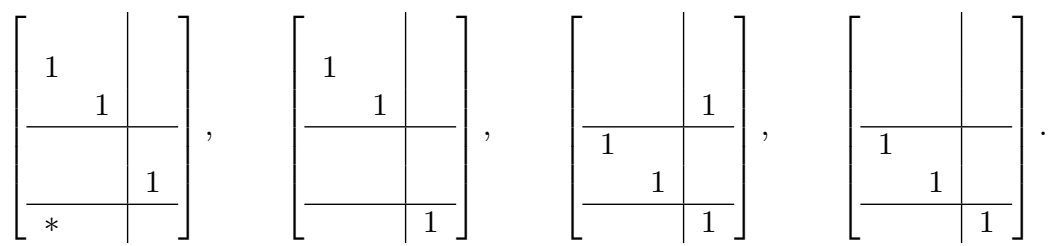

Therefore, in $\operatorname{Inv}(p, q)$ there are four marked classes and only one non-marked class $\tilde{Y}$ having marked type $Y(3,2,1)$ and PM reduced form

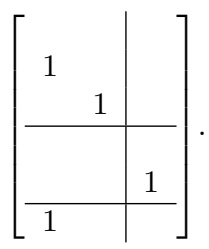

Remark 6.2 In addition, we can compute easily the Segre characteristic of the quotient endomorphism in each case: $(1,1,1)$ for $Y \widetilde{(3,2,1)} ;(2,1)$ for $\overline{Y(3,1,2)}, \widetilde{Y(2,3,1)}$ and $\tilde{Y}$; and $(3)$ for $Y \widetilde{(2,1,3)}$.

In particular, we conclude that the Carlson problem has 3 solutions in this case, so that the Segre characteristic of the quotient is not sufficient to classify in Inv $(p, q)$.

In this case the Littlewood Richardson sequences (see [3]) do not provide the classification neither. Indeed, for the three cases with the same Segre characteristic of the quotient, the LR sequences are: (2,1), (3,1,1), (3,2,1) for $\widehat{Y(3,1,2)}$; (2,1), (3,2), (3,2,1) for both $\widehat{Y(2,3,1)}$ and $\tilde{Y}$.

\subsection{Monogenic subspaces}

For monogenic subspaces (that is, $m=1$ ) the classification problem was solved in [11] and [4]. Indeed, the last one motivated the present work. If $m=1$, the vectorial representations in $B L D^{*}\left(p, q_{1}\right)$ are simply block columns

$$
Y=Y_{* 1}, \quad Y_{i, 1} \in M_{p_{i} \times q_{1}}
$$

which in [4] are represented by its first column (the "generator" vector). The marked type is reduced to a simple index $p_{i_{1}}$ (in [4] $p_{i_{1}}-q_{1}$ is called the "marked" index), so that in the reduced PM forms:

$$
Y_{i_{1}, 1}=I_{i_{1}, 1}^{q_{1}}, \quad Y_{i 1}=0 \quad \text { if } \quad i<i_{1}
$$


Let $i(2)<\ldots<i(r)$ be the row indices of the remainder non-zero blocks in $Y_{* 1}$ : $Y_{i, 1} \neq 0 \quad$ iff $\quad i=i_{1}, i(2), \ldots, i(r)$.

Clearly, by means of row BLD-ETs, the non-zero blocks can be reduced to unitary ones $Y_{i(s), 1}=$ $I_{i(s), 1}^{k(s)}$, where $k(s)=h_{i(s), 1}$, for $2 \leq s \leq r$.

Therefore, in this particular case we have PM canonical forms, which classify the monogenic subspaces, each class being determined by the indices

$$
i_{1} ; \quad i(s), k(s), \quad 2 \leq s \leq r
$$

Notice that $p_{i(2)}-p_{i_{1}}+q_{1}<k(2)<q(1)$ so that $p_{i(2)} \leq p_{i_{1}}-2$ and analogously for $p_{i(3)}, \ldots$. Hence, we have the next proposition equivalent to proposition 5.4 in [4]:

Proposition 6.3 [4] Let $\mathcal{V}$ be a monogenic subspace. We can assume that it belongs to Inv $\left(p, q_{1}\right)$.

(1) The marked type is characterized by a unique marked index $1 \leq i_{1} \leq n$.

(2) For any marked type, each equivalence class has an unique PM reduced form, having at maximum a non-zero parameter (which can be valued 1) in the blocks $Y_{i, 1}, i>i_{1}$, that is to say, each class has a PM canonical form characterized by $i_{1}<i(2)<\cdots<i(r) \leq n$ and $1 \leq k(r)<\ldots<k(2)<q_{1}$, as follows:

$Y_{i, 1}=0$ iff $i \neq i_{1}, i(2), \ldots, i(r) ; \quad Y_{i_{1}, 1}=I_{i_{1}, 1}^{q_{1}}$ and $Y_{i(s), 1}=I_{i(s), 1}^{k(s)}, \quad$ for $2 \leq s \leq r$.

(3) The sets of possible values $i(s)$ and $k(s)$, for $2 \leq s \leq r$ are characterized by:

(i) $p_{i(2)} \leq p_{i_{1}}-2, p_{i(3)} \leq p_{i(2)}-2, \ldots$

(ii) $q_{1}>k(2)>p_{i(2)}-p_{i_{1}}+q_{1}, k(2)>k(3)>p_{i(3)}-p_{i(2)}+k(2), \ldots$

(3') In particular, if we restrict ourselves to the indecomposable subspaces (that is to say: $\left.i_{1}=1, i(s)=s\right)$ we have:

(i) $p_{2} \leq p_{1}-2, p_{3} \leq p_{2}-2, \ldots$

(ii) $q_{1}>k(2)>p_{2}-p_{1}+q_{1}, k(2)>k(3)>p_{3}-p_{2}+k(2), \ldots$

Example 6.4 The (non marked) indecomposable monogenic classes for $q_{1}=2$ or 3 are:

$$
\left(\begin{array}{cc}
\cdot & \cdot \\
1 & 0 \\
0 & 1 \\
\hline \cdot & \cdot \\
1 & 0
\end{array}\right) \quad\left(\begin{array}{ccc}
\cdot & \cdot & \cdot \\
1 & 0 & 0 \\
0 & 1 & 0 \\
0 & 0 & 1 \\
\hline \cdot & \cdot & \cdot \\
1 & 0 & 0 \\
0 & 1 & 0
\end{array}\right) \quad\left(\begin{array}{ccc}
\cdot & \cdot & \cdot \\
1 & 0 & 0 \\
0 & 1 & 0 \\
0 & 0 & 1 \\
\hline \cdot \cdot & \cdot & \cdot \\
1 & 0 & 0
\end{array}\right) \quad\left(\begin{array}{ccc}
\cdot & \cdot & \cdot \\
1 & 0 & 0 \\
0 & 1 & 0 \\
0 & 0 & 1 \\
\hline \cdot & \cdot & \cdot \\
1 & 0 & 0 \\
0 & 1 & 0 \\
\hline \cdot & \cdot & \cdot \\
1 & 0 & 0
\end{array}\right)
$$

where the dots mean additional null rows in such a way that $p_{2} \leq p_{1}-2, p_{3} \leq p_{2}-2$. 


\subsection{Degree of the minimal polynomial lower than 6}

On the other hand, in [12] one shows that the number of classes is finite if and only if the degree of the minimal polynomial is lower than 6 . Here, by means of PM vectorial reduced forms, we study the indecomposable non-monogenic invariant subspaces in $\operatorname{Inv}(p, q)$, with $p_{1} \leq 5$. Indeed, for each possible PM reduced form, we will separate successively the indecomposable invariant components by applying corollary 5.7. As the first consequence we obtain the following necessary conditions for the indecomposable non-monogenic invariant subspaces:

Proposition 6.5 Let $Y_{\text {red }}\left(p^{\prime}\right)(*)$ be a PM reduced form of an indecomposable non-monogenic invariant subspace. Then,

(1) $p_{i_{j}}>q_{j}$ for all $1 \leq j \leq m$,

(2) $q_{1}>2$.

Proof.

(1) By construction $p_{i_{j}} \geq q_{j}$. But $p_{i_{j}}=q_{j}$ is not possible because of corollary 5.7.

(2) Again, it is a direct consequence of corollary 5.7.

Now, we can tackle the main result:

Theorem 6.6 Let us consider Inv $(p, q)$, with $p_{1} \leq 5$. Then, there are only 14 indecomposable non-monogenic invariant subspaces:

(i) For $p_{1}=1,2$ or 3 there are no indecomposable non-monogenic invariant subspaces.

(ii) For $p_{1}=4$, the only indecomposable non-monogenic invariant subspace is

$\left[\begin{array}{lll|l}0 & 0 & 0 & \\ 1 & 0 & 0 & \\ 0 & 1 & 0 & \\ 0 & 0 & 1 & \\ \hline 1 & 0 & 0 & 0 \\ 0 & 1 & 0 & 1\end{array}\right]$

(iii) For $p_{1}=5$ and $q_{1}=4$, there are 7 indecomposable non-monogenic invariant subspaces:

(1)

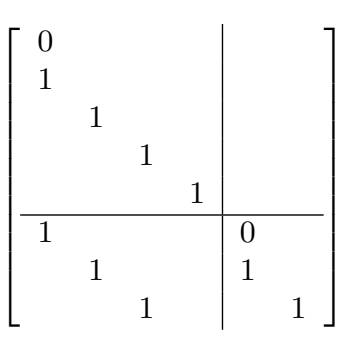

,$(2)$

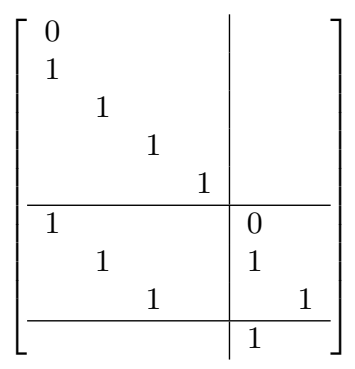

,(3)

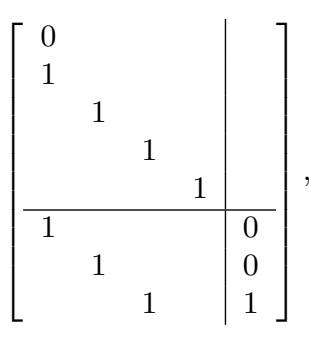

, (4)

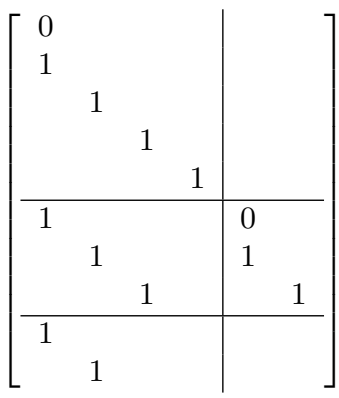




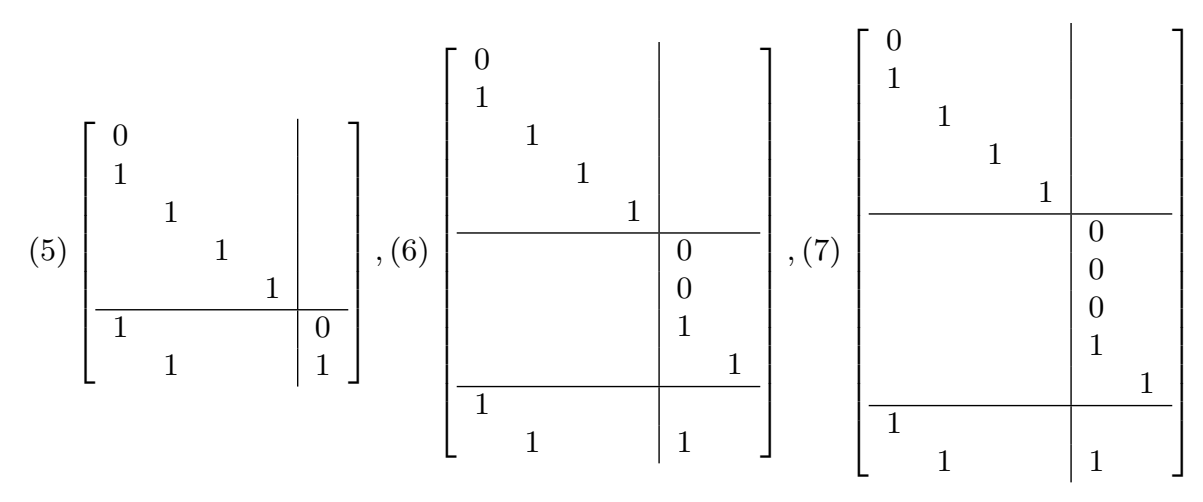

(iv) For $p_{1}=5$ and $q_{1}=3$, there are 6 indecomposable non-monogenic invariant subspaces:

(8)

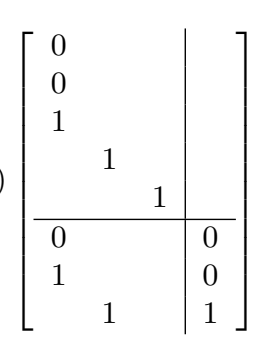

,$(9)$

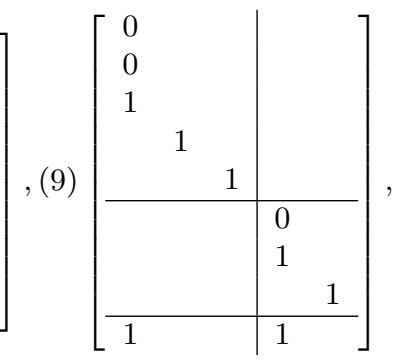

(11)

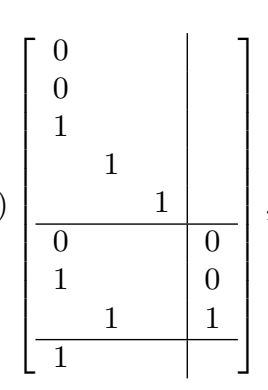

$(12)$
(10)
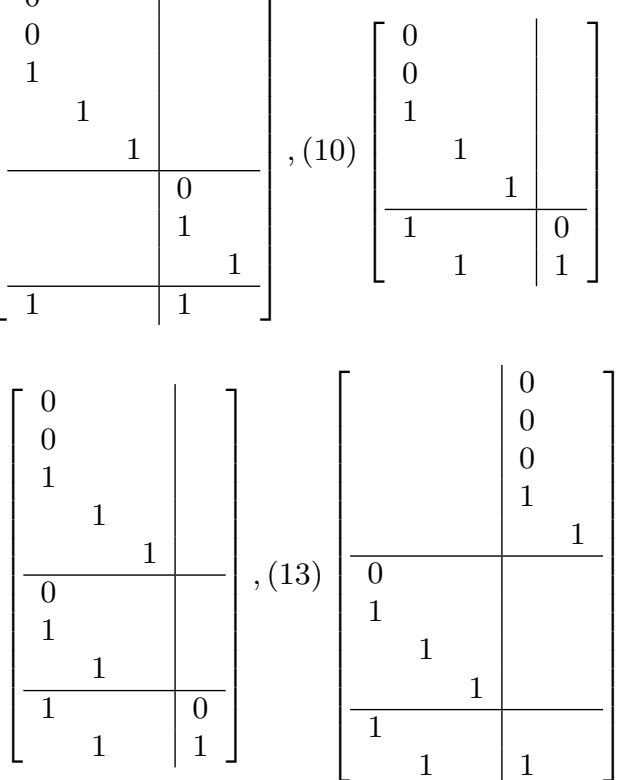

,(13)

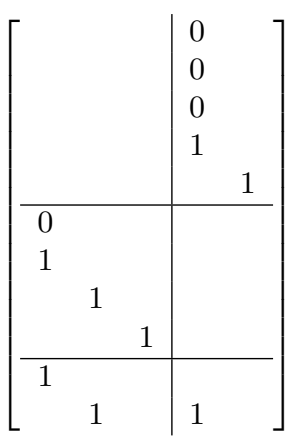

Proof.

In all the proof $M(s, l ; k) \in M_{s, l}(\mathbb{C})$ will be a lower diagonal matrix with the only non-zero entries in the $k$-diagonal (see (4) in definition 2.10).

(i) It is obvious from the above proposition.

(ii) For the given invariant subspace, it is clear that none BLD-ET can eliminate any entry, so that it is indecomposable.

Let us see that, when $p_{1}=4$, any invariant subspace has an invariant component of the above type or a monogenic invariant component with $p_{1}=4$. Thus, by recurrence, we return to the case $p_{1} \leq 3$ above.

We know that any invariant subspace is monogenic or decomposable if $q_{1} \leq 2$ or $p_{i_{j}}=q_{j}$. Therefore, let us consider $Z \equiv Y_{\text {red }}\left(p^{\prime}\right)(*)$ be a generic PM reduced form of an invariant subspace with $q_{1}=3$ and $p_{i_{1}}=p_{1}=4$, that is to say, $Z_{11}=I_{11}^{3} \in M_{4,3}(\mathbb{C})$ is a main 
unitary block. Also, if $q_{j}=3$ and $p_{i_{j}}=4$, then $Z_{i_{j}, j}=I_{i_{j}, j}^{3}$ and the possible non-zero parametrised blocks in $Z_{* j}$ are $M(2,3 ; 2)$ or $M(1,3 ; 1)$.

If $q_{j}=2$ and $p_{i_{j}}=4$, then $Z_{i_{j}, j}=I_{i_{j}, j}^{2}$ and the possible non-zero parametrised blocks in $Z_{* j}$ are $M(2,2 ; 1)$ or $M(1,2 ; 1)$.

If $q_{j}=2$ and $p_{i_{j}}=3$, then $Z_{i_{j}, j}=I_{i_{j}, j}^{2}$ and the possible non-zero parametrised blocks are $M(1,2 ; 1)$.

If $q_{j}=1$, only the unitary block is non-zero in $Z_{* j}$.

We will proceed by recurrence when some block $Z_{i 1}, i \neq 1$, is non-zero. Else, $S p\left(Z_{* 1}\right)$ is a (marked) monogenic invariant component.

Firstly, let us assume that there is some non-zero $Z_{i 1}=M(2,3 ; 2)$ in a row block which does not contain any unitary block, that is to say $i \neq i_{j}$ for all $1 \leq j \leq m$. Then, by means of $(i, 1)$-row BLD-ET, the other blocks $Z_{k 1}=M(2,3 ; 2)$ or $Z_{k 1}=M(1,3 ; 1)$ become 0 . Also, by means of $(i, 1)$-column BLD-ET, the blocks $Z_{i j}, j>1$ become 0; notice that the main unitary blocks $Z_{i_{j}, j}$ do not change and that the perturbed blocks $Z_{1 j}$ can be returned to 0 by means of $\left(i_{j}, j\right)$-row BLD-ETs (that does not change the other blocks because in this case $Z_{i_{j}, k}=0,1 \leq j \leq m$ for $k \neq j$ ). Hence, in this case, we have obtained a PM reduced form with $Z_{i_{1}, 1}=I_{i_{1}, 1}^{3}$ and $Z_{i 1}=M(2,3 ; 2)$ the only two non-zero blocks in $Z_{* 1}$ and $Z_{i_{1}, j}=Z_{i j}=0$ for $j>1$. Then, $S p\left(Z_{* 1}\right)$ is a (non-marked) monogenic invariant component.

Secondly, let us assume that there are no non-zero blocks $Z_{i 1}=M(2,3 ; 2)$ with $i \neq i_{j}$ for all $1 \leq j \leq m$, but there is some non-zero block $Z_{i_{j}, 1}=M(2,3 ; 2)$. As $p_{i_{j}}=2$, it must be $q_{j}=1$, so that $Z_{i_{j}, j}=I_{i_{j}, j}^{1} \in M_{2,1}(\mathbb{C})$. Again the other blocks in $Z_{i_{j} *}$ become 0 by means of $\left(i_{j}, 1\right)$-column BLD-ET. Also the blocks $Z_{k 1}=M(1,3 ; 1)$ by means of $\left(i_{j}, 1\right)$-row BLD-ET. Notice that one uses only the up row of $Z_{i_{j}, 1}$, so that no change is induced in $Z_{* j}$. Finally, the other blocks $Z_{k 1}=M(2,3 ; 2)$ become 0 by means of $\left(i_{j}, 1\right)$-row BLD-ET, and the changes in $Z_{k j}$ can be reversed by column BLD-ETs with the unitary block in $Z_{k *}$ (also belonging to $M_{3,1}(\mathbb{C})$ ). Hence, $S p\left(Z_{* 1}\right) \oplus S p\left(Z_{* j}\right)$ is a non-monogenic invariant component: the announced one.

Thirdly, let us assume that there are no non-zero blocks $Z_{i 1}=M(2,3 ; 2)$, but there is $Z_{i 1}=$ $M(1,3 ; 1)$ for some $i$. In this case none unitary block is possible in $Z_{i *}$. By means of $(i, 1)$ row BLD-ET we obtain a new PM reduced form where the other blocks $Z_{k 1}=M(1,3 ; 1)$ become 0 . Next, by means of $(i, 1)$-column BLD-ET the blocks $Z_{i j}=M(1,3 ; 1), j>1$ become 0 . If there are no non-zero blocks $Z_{i j}=M(1,2 ; 1)$, this new PM reduced form shows that $\operatorname{Sp}\left(Z_{* 1}\right)$ is a (non-marked) monogenic invariant component.

Fourthly, if there is some non-zero block $Z_{i j}=M(1,2 ; 1), p_{i_{j}}=4$, the other $Z_{k j}=$ $M(1,2 ; 1), k \neq i$ become 0 by means of $(i, j)$-row BLD-ET. Next, by means of $(i, j)$-column BLD-ET followed by $\left(i_{s}, s\right)$-row BLD-ET for $s \neq j, S p\left(Z_{* j}\right)$ becomes a monogenic invariant component. Repeating this elimination for the other $Z_{i s}=M(1,2 ; 1), p_{i_{s}}=4$ we come back to the third case.

(iii) We sketch the proof following the same recurrent reasoning. Now, for $Z \equiv Y_{\text {red }}\left(p^{\prime}\right)$, we assume $q_{1}=4$ and $p_{i_{1}}=p_{1}=5$, that is, $Z_{11}=I_{11}^{4} \in M_{5,4}(\mathbb{C})$. The other possible non-zero blocks $Z_{i 1}, i \neq 1$, are $M(3,4 ; 3), M(2,4 ; 2)$ or $M(1,4 ; 1)$.

In the first case, the only possible non-zero blocks $Z_{i j}, j \neq 1$, are the unitary ones of the type $M(3,2 ; 2)$ or $M(3,1 ; 1)$. If the remainder blocks in $Z_{* 1}$ are 0 , then we obtain irreducible non-monogenic components of the form (1) and (2) in the first case, or of the 
form (3) in the second case. If there is in $Z_{* 1}$ a non-zero block of the form $M(2,4 ; 2)$, it can be made 0 by means of row BLD-ETs, giving the case (4)(in its PM reduced form). If it is of the form $M(1,4 ; 1)$, it can be also made 0 without additional changes.

Secondly, let us assume that in $Z_{* 1}$ there is some non-zero block of the form $M(2,4 ; 2)$ and none of the form $M(3,4 ; 3)$. As above the non-zero blocks of the form $M(1,4 ; 1)$ can be made 0 without additional changes. Only two types of non-zero blocks are possible in $Z_{i *}$ : $M(2,1 ; 1)$, which gives $(5) ; M(2,2 ; 1)$, which gives (6) or $(7)$.

Thirdly, if in $Z_{* 1}$ there are only additional non-zero blocks of the form $M(1,4 ; 1)$, then $\operatorname{Sp}\left(Z_{* 1}\right)$ is a monogenic component.

(iv) In a similar way, if $q_{1}=3$ and $p_{i_{1}}=p_{1}=5$ that is, $Z_{11}=I_{11}^{3} \in M_{5,3}(\mathbb{C})$, the other possible non-zero blocks $Z_{i 1}, i \neq 1$, are $M(2,3 ; 2), M(3,3 ; 2)$ or $M(1,3 ; 1)$ and we obtain the cases (8), (9), (10), (11) and (12). If $q_{1}=3$ and $p_{i_{1}}=p_{2}=4$ that is, $Z_{21}=I_{21}^{3} \in M_{4,3}(\mathbb{C})$, we obtain (13).

\subsection{More general cases}

For $p_{1}=6$ and $q_{2} \neq 0$, continuous valued parameters, giving different equivalence classes for each value, can appear. For example:

\section{Proposition 6.7 Let}

$$
Y_{x}=\left[\begin{array}{llll|ll}
0 & 0 & 0 & 0 & 0 & 0 \\
0 & 0 & 0 & 0 & 0 & 0 \\
1 & 0 & 0 & 0 & 0 & 0 \\
0 & 1 & 0 & 0 & 0 & 0 \\
0 & 0 & 1 & 0 & 0 & 0 \\
0 & 0 & 0 & 1 & 0 & 0 \\
\hline 0 & 0 & 0 & 0 & 0 & 0 \\
1 & 0 & 0 & 0 & 0 & 0 \\
0 & 1 & 0 & 0 & 1 & 0 \\
0 & 0 & 1 & 0 & 0 & 1 \\
\hline 1 & 0 & 0 & 0 & 0 & 0 \\
0 & 1 & 0 & 0 & x & 0
\end{array}\right] \in B L D^{*}((6,4,2),(4,2))
$$

Then, $S p\left(Y_{x}\right)$ and $S p\left(Y_{y}\right)$ are not equivalent if $x \neq y$.

Proof.

Let us see that $S p\left(Y_{x}\right)$ and $S p\left(Y_{y}\right)$ are not equivalent if $x \neq y$. That is to say that there are no $A=\left[\begin{array}{lll}A_{11} & A_{12} & A_{13} \\ A_{21} & A_{22} & A_{23} \\ A_{31} & A_{32} & A_{33}\end{array}\right] \in B L D^{*}(p, p), B=\left[\begin{array}{cc}B_{11} & B_{12} \\ B_{21} & B_{22}\end{array}\right] \in B L D^{*}(q, q)$ such that $A Y_{x}=Y_{y} B$.

Taking into account that $Y_{x}=\left[\begin{array}{cc}I_{11}^{4} & 0 \\ I_{21}^{3} & I_{22}^{2} \\ I_{31}^{2} & x I_{32}^{1}\end{array}\right] \in B L D^{*}(p, q)$ (see definition 2.10), the above system splits into the matricial equations: 


$$
\begin{aligned}
A_{11} I_{11}^{4}+A_{12} I_{21}^{3}+A_{13} I_{31}^{2} & =I_{11}^{4} B_{11} \\
A_{12} I_{22}^{2}+x A_{13} I_{32}^{1} & =I_{11}^{4} B_{12} \\
A_{21} I_{11}^{4}+A_{22} I_{21}^{3}+A_{23} I_{31}^{2} & =I_{21}^{3} B_{11}+I_{22}^{2} B_{21} \\
A_{22} I_{22}^{2}+x A_{23} I_{32}^{1} & =I_{21}^{3} B_{12}+I_{22}^{2} B_{22} \\
A_{31} I_{11}^{4}+A_{32} I_{21}^{3}+A_{33} I_{31}^{2} & =I_{31}^{2} B_{11}+y I_{32}^{1} B_{21} \\
A_{32} I_{22}^{2}+x A_{33} I_{32}^{1} & =I_{31}^{2} B_{12}+y I_{32}^{1} B_{22}
\end{aligned}
$$

In particular, we have $a_{22}^{4}=b_{11}^{4}, a_{22}^{4}=b_{22}^{2}, a_{33}^{2}=b_{11}^{4}$ and $x a_{33}^{2}=y a_{22}^{4}$ from (3), (4), (5) and (6) respectively. As $a_{33}^{2}, b_{22}^{2} \neq 0$ because $A_{33}$ and $B_{22}$ have maximal rank, it implies $x=y$.

\section{A geometrical interpretation}

Here we present a geometrical point of view of our constructions. It is not necessary for the validity of our results and conclusions, but perhaps it makes them more clear and motivated.

\subsection{Geometric structures}

First, we reformulate the conclusions in section 2.2 .

Corollary 7.1 Let $(p, q)$ and $J_{p}$ be as in definition 2.7.

1. The map $\varphi: B L D^{*}(p, q) \rightarrow \operatorname{Inv}_{q}\left(J_{p}\right), \quad \varphi(Y)=S p(Y)$ is surjective and factorizes as follows:

$$
B L D^{*}(p, q) \rightarrow B L D^{*}(p, q) / B L D^{*}(q, q) \cong \operatorname{Inv}_{q}\left(J_{p}\right) \subset \operatorname{Inv}(p, q)
$$

2. It induces a bijection

$$
B L D^{*}(p, q) / \sim \cong \operatorname{Inv}(p, q) / \sim
$$

where we recall that: $\tilde{Y}=\left\{P Y Q: P \in B L D^{*}(p, p), Q \in B L D^{*}(q, q)\right\} \subset B L D^{*}(p, q)$, $\left(S \widetilde{p(Y)}, J_{p}\right)=\left\{S p\left(S^{-1} Y\right), S^{-1} J_{p} S: S \in M_{N}(\mathbb{C})\right\} \subset \operatorname{Inv}(p, q)$.

Explicitly: $\tilde{Y}=\varphi^{-1}\left(\operatorname{Inv}_{q}\left(J_{p}\right) \cap\left(\widetilde{S p(Y)}, J_{p}\right)\right.$.

In addition, differentiable structures can be considered in the above sets. In order to do that, we consider the set $G r_{N}(d) \times M_{N}(\mathbb{C})$ of general pairs $(\mathcal{V}, A)$ where $G r_{N}(d)$ is the Grassmannian of $d$-dimensional subspaces of $\mathcal{V} \in E$. Hence

$$
\operatorname{Inv}(p, q) \subset \operatorname{Inv}_{d}(E) \subset G r_{N}(d) \times M_{N}(\mathbb{C}) .
$$

We start with $\operatorname{Inv}_{d}(E)$ :

Proposition 7.2 [7] We have that: 
(1) Inv $_{d}(E)$ is a submanifold of $G r_{N}(d) \times M_{N}(\mathbb{C})$ having dimension $N^{2}$.

(2) Each class $\widetilde{(\mathcal{V}, A)}$ is a submanifold. Indeed, it is an orbit with regard to the action of the Lie group $M_{N}^{*}(\mathbb{C}): \quad \widetilde{(\mathcal{V}, A)}=\left\{\left(S^{-1} \mathcal{V}, S^{-1} A S\right), \quad S \in M_{N}^{*}(\mathbb{C})\right\}$.

In [8] one shows that $\operatorname{Inv}_{d}\left(J_{p}\right)$ is not, in general, a submanifold but it is a stratified manifold: $\operatorname{Inv} v_{d}\left(J_{p}\right)=\cup_{q} \operatorname{Inv} v_{q}\left(J_{p}\right)$.

Proposition 7.3 [8] We have that:

(1) The quotient space $\operatorname{Inv}_{q}\left(J_{p}\right) \cong B L D^{*}(p, q) / B L D^{*}(q, q)$ has a differentiable structure such that the map $\varphi$ in the corollary 7.1 is a submersion.

(2) Each class $\tilde{Y}$ is a submanifold. Indeed, an orbit with regard to the action of a Lie group:

$$
\tilde{Y}=\left\{P Y Q, \quad P \in B L D^{*}(p, p), Q \in B L D^{*}(q, q)\right\}
$$

Its dimension has been computed in [5] when it is marked.

\subsection{Miniversal deformations}

These differentiable structures allow us to use Arnold's techniques ([1], [8] for the basic concepts and results) in order to study the classes near a given one $\widehat{(\mathcal{V}, A)}$ or equivalently near $\tilde{Y}$. The starting point is that any invariant subspace is a perturbation of a marked one (see [6]), not unique in general. In turn, Arnold's theory warrants that any perturbation of an object is represented in the so-called miniversal deformation: a (minimally) parametrised family of objects, centered in the initial one. Thus, any invariant subspace appears in the miniversal deformation of some marked subspace. For such a subspace, the miniversal deformation of its canonical vectorial representation has been obtained in [5].

Example 7.4 For the marked subspaces in example 2.20, the miniversal deformations in [5] are respectively:
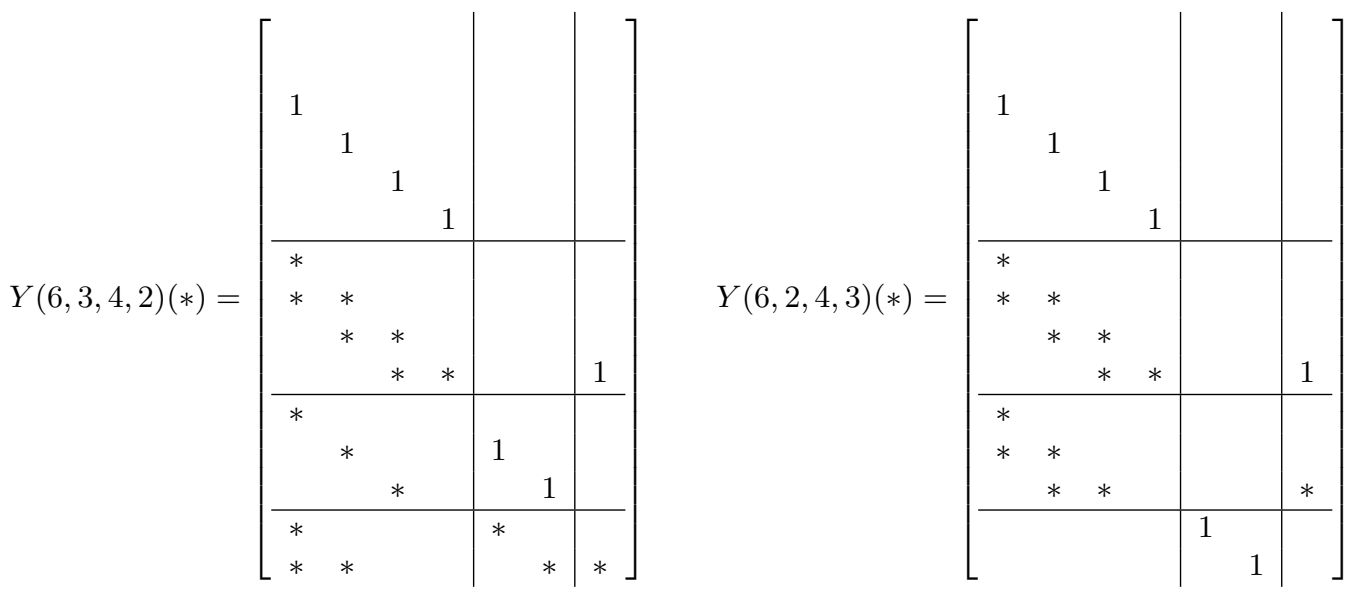
However, an invariant subspace can be represented in several of these miniversal deformations. For example it can be seen that in $\operatorname{Inv}((3,2,1),(2,1,0))$, the invariant subspace $Y(3,2,1)$ is represented in the miniversal deformations of all marked subspaces. To avoid this redundance, we will restrict the parameters in the miniversal deformation in [5] in order to each invariant subspace is represented in only one of them.

Indeed, let us see that the PM reduced families in definition 5.4 are just these desired restricted miniversal deformation.

Proposition 7.5 Let $Y\left(p^{\prime}\right) \in B L D^{*}(p, q)$ be the canonical representation of a marked subspace (see definition 2.19). Then, the miniversal deformation of $Y\left(p^{\prime}\right)$ in [5] can be obtained by applying the BLD-ETs in definitions 3.2 and 3.5 to a general perturbation $Y\left(p^{\prime}\right)(*)$ in $B L D^{*}(p, q)$.

\section{Proof.}

The proof has the same four steps as in the proof of theorem 5.3 and it is very analogous to it. We have take into account that in all the transformations the non nullity of the diagonals $D_{i_{1}, 1}^{q_{1}}, \ldots D_{i_{m}, m}^{q_{m}}$ is not affected because we assume that the parameters of the perturbation are small, and they can become equal to 1 by multiplying by a factor near to 1 .

The next example shows the sequence of BLD-ETs which makes 0 as many perturbation parameters $(*)$ as possible.

Example 7.6 Let us consider the marked subspace represented by $Y \in B L D^{*}((6,4,3,2),(4,2,1))$, where $Y=Y(6,3,4,2)$, and a general perturbation $Y(*)$, where the new entries are assumed much smaller than 1. Applying (1) as in theorem 5.3 or in definition 5.4 we obtain $Y_{1}(*)$.

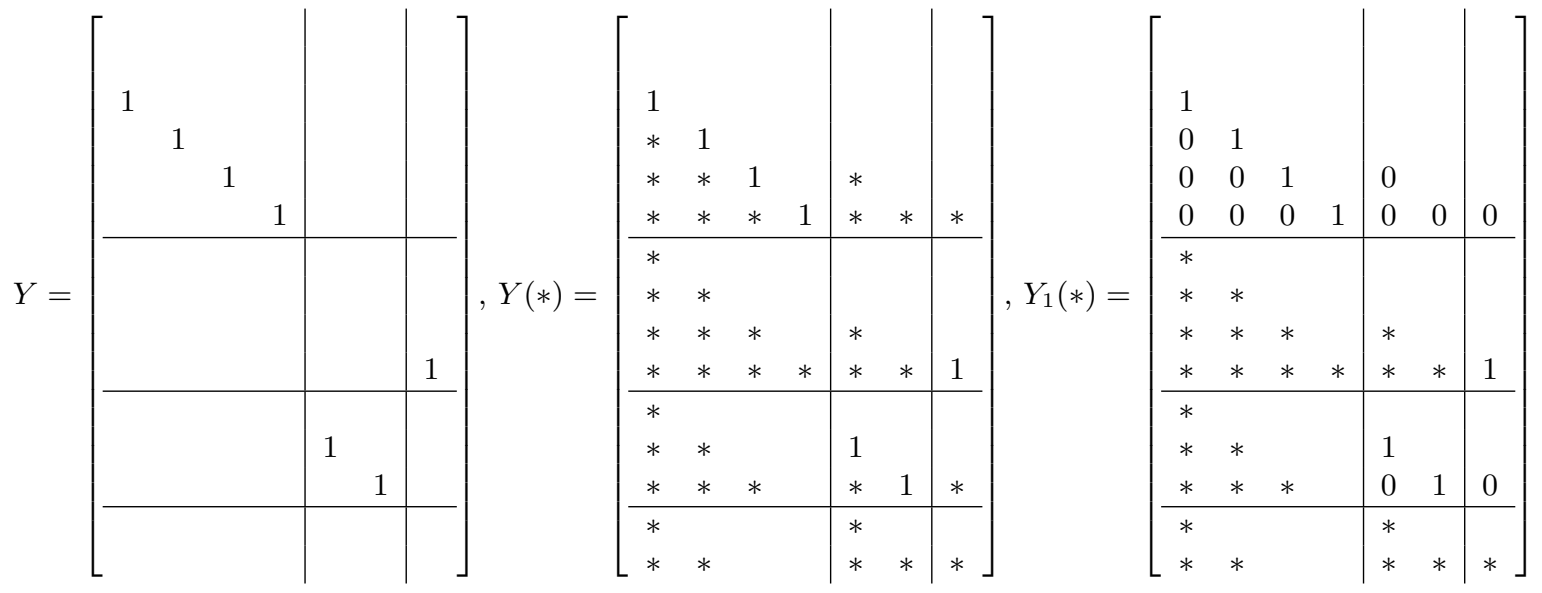

Applying (2), (3) and (4) as in theorem 5.3 or in definition 5.4 successively we obtain $Y_{2}(*)$, $Y_{3}(*)$ and $Y_{4}(*)$. This last is the desired miniversal deformation of $Y$ in [5]. 


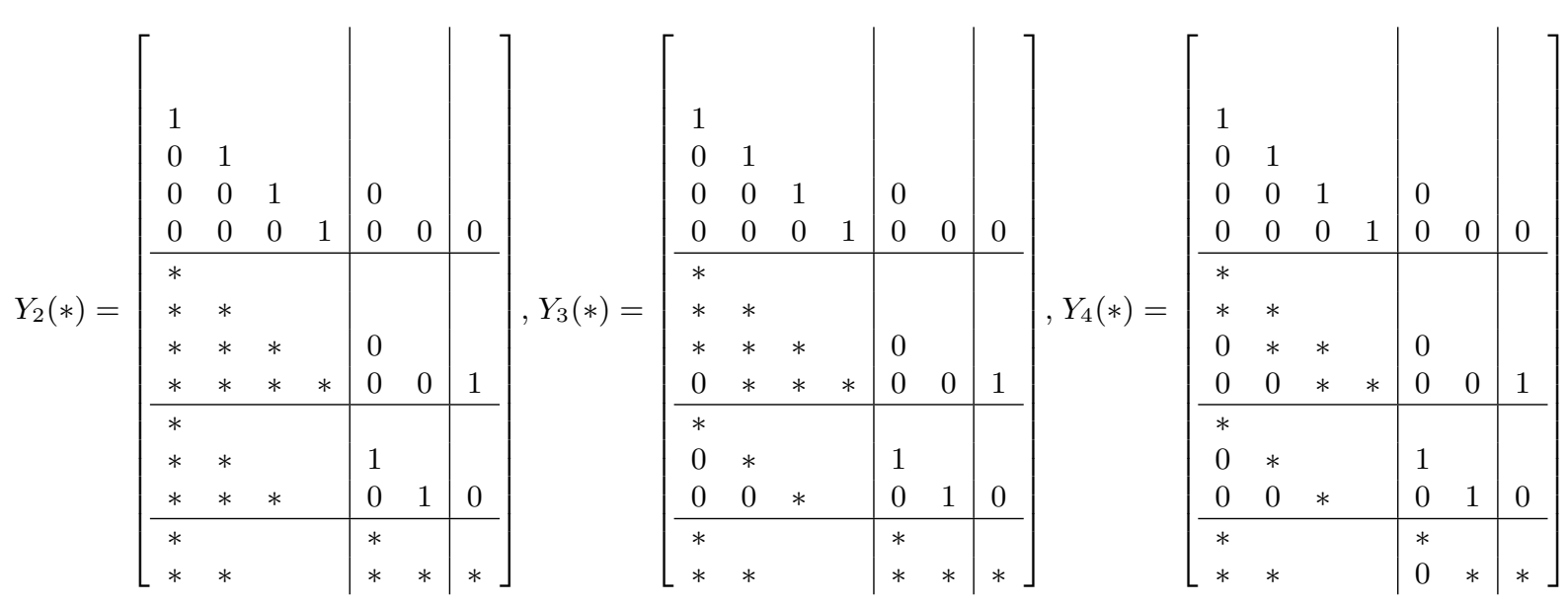

Thus, as announced, we have:

Corollary 7.7 (1) The PM reduced family $Y_{\text {red }}\left(p^{\prime}\right)(*)$ is the subfamily of the miniversal deformation of $Y\left(p^{\prime}\right)$ in [5] such that the main diagonals other than $D_{i_{j} 1}^{q_{j}}$ are 0 . In this way the marked subspaces other than the central one $Y\left(p^{\prime}\right)$ are eliminated.

(2) The number of parameters that appear in the miniversal deformation in [5] is reduced to:

$$
\sum_{1 \leq j \leq m} \sum_{s>j} \max \left(0, \min \left(p_{i_{j}}-p_{i_{s}}-1, q_{j}-q_{s}-1, p_{i_{j}}-q_{j}, p_{i_{s}}-q_{s}\right)\right) .
$$

Proof.

(1) It is straightforward.

(2) Counting accurately the parameters that appear in theorem 5.3 we obtain:

$$
\sum_{1 \leq j \leq m} \sum_{s>j} \max \left(0, \min \left(q_{j}-1, p_{i_{s}}\right)-\max \left(p_{i_{s}}-p_{i_{j}}+q_{j}, q_{s}\right)\right)
$$

and studying the four possible cases we can see that this number is the same as the one in the statement.

\subsection{Marked perturbations}

We have seen in section 7.2 that any invariant subspace appears in one and only in one restricted miniversal deformation (PM reduced family). Its center is just its marked type in section 4 . There, it has been obtained by means of the $\mathrm{M}$ algorithm. We can see that once again it has a geometrical interpretation: for any invariant subspace $\mathcal{V}=S p(Y)$, its "marked type" is, among the marked subspaces lying in the boundary of $\tilde{Y}$, the one whose orbit has the maximal dimension.

In order to see that, given a marked subspace, we study its perturbations which are also marked. They can be studied starting from the miniversal deformations in [5]. However, we present an autonomous approach. 
Definition 7.8 Let $\mathcal{V} \in \operatorname{Inv}(p, q)$ be a marked subspace and $Y \equiv Y\left(p^{\prime}\right) \in B L D^{*}(p, q)$ be its canonical vectorial representation (see definition 2.19). Its marked uniparametric perturbations are the marked subspaces obtained by introducing a unique non-zero additional parameter in $Y$.

Example 7.9 Let us consider $p=(6,4,3,2), q=(4,2,1)$ and $Y=Y(6,3,4,2)$ as in example 7.6. It is clear that the only parameters which can give marked uniparametric perturbations are those placed in the main diagonals: $\alpha_{1}, \ldots \alpha_{7}$

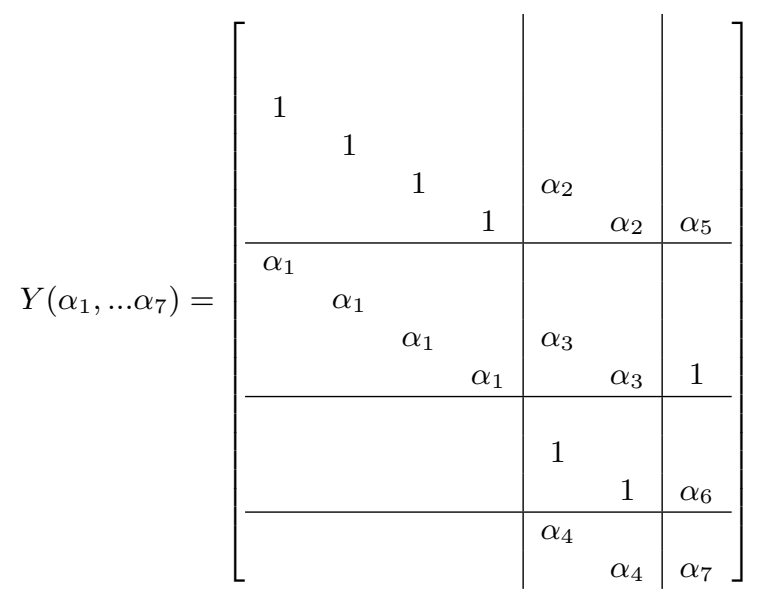

Clearly $\alpha_{2}, \alpha_{3}, \alpha_{5}$ can be eliminated by means of row BLD-ETs, so that, the corresponding invariant subspaces are equivalent to $Y$. Analogously $\alpha_{6}$ can be eliminated by means of column BLD-ETs.

However, if $\alpha_{1} \neq 0$, the blocks $Y_{11}$ and $Y_{23}$ can be made 0 , whereas $Y_{13}$ becomes non-zero. Hence $Y\left(\alpha_{1}\right) \cong Y(4,3,6,2)$. Analogously, $Y\left(\alpha_{4}\right) \cong Y(6,2,4,3), \quad Y\left(\alpha_{7}\right) \cong Y(6,3,2,4)$.

Notice that in the above example the new permutation of $p$ is obtained by means of a transposition in the initial one. In addition, the new transposed pairs are increasing: $(4,6),(2,3),(2,4)$. The following theorem shows that it is so in general.

Definition 7.10 Let us consider a permutation $\left(p_{i_{1}}, \ldots p_{i_{n}}\right)$ of $p$ compatible with $q$. The number of inversions is the number of pairs $p_{i_{j}}, p_{i_{j+r}}$ such that $p_{i_{j}}<p_{i_{j+r}}, 1 \leq i_{j}, i_{j+r} \leq n$. We call a compatible inverse transposition any new permutation of $p$ compatible with $q$ which differs only in permuting a pair $p_{i_{j}}, p_{i_{j+r}}$ such that $p_{i_{j}}>p_{i_{j+r}}$. Therefore, the number of inversions of the new permutation is greater than before.

Theorem 7.11 Given a permutation $p^{\prime}=\left(p_{i_{1}}, \ldots, p_{i_{n}}\right)$ of $p$ compatible with $q$, then there is a one-to-one correspondence between the compatible inverse transpositions of $p^{\prime}$ and the marked uniparametric perturbations of $Y\left(p^{\prime}\right)$.

Proof. Let us assume $p_{i_{j}}>p_{i_{j+r}}$ and that the corresponding inverse transposition $\bar{p}$ is also compatible with $q$. Let us consider the uniparametric perturbation $Y(\alpha)$ obtained from $Y \equiv$ $Y\left(p^{\prime}\right)$ by means of a parameter $\alpha \neq 0$ placed in the main diagonal $D_{i_{j+r}, j}^{q_{j}}$. Let us see that $Y(\alpha)$ is equivalent to $Y(\bar{p})$ : clearly $\bar{p}_{i_{j}}=p_{i_{j+r}}$ and by means of a row BLD-ET with $D_{i_{j+r}, j}^{q_{j}}$ we can 
make 0 the block $Y_{i_{j}, j}$; it is enough if $Y_{i_{j}, j+r}=0$ (that is $q_{j+r}=0$, as for $\alpha_{4}$ and $\alpha_{7}$ in the above example); if it is not (as for $\alpha_{1}$ in the above example), we can make $Y_{i_{j}, j+r}=0$ by means of a column BLD-ET again with $D_{i_{j+r}, j}^{q_{j}}$. In any case, $\quad Y(\alpha) \cong Y(\bar{p})$.

Conversely, starting on $Y \equiv Y\left(p^{\prime}\right)$, a uniparametric perturbation $Y(\alpha), \alpha \neq 0$, is marked if the parameter $\alpha$ is placed in a main diagonal, say $D_{i_{j+r}, j}^{q_{j}}$. Indeed, if $p_{i_{j}}<p_{i_{j+r}}$, the parameter $\alpha$ can be made 0 by means of a row BLD-ET with $D_{i_{j}, j}^{q_{j}}$, so that $Y(\alpha) \cong Y$. On the other hand, if $p_{i_{j}}>p_{i_{j+r}}$, then, proceeding as above, it results that $Y(\alpha) \cong Y(\bar{p})$.

This theorem leads to a natural organization of the marked subspace in $\operatorname{Inv}(p, q)$ as an oriented graph:

Definition 7.12 The marked subspaces in $\operatorname{Inv}(p, q)$ can be organized as an oriented graph as follows:

(1) The vertices are the permutations of $p$ compatible with $q$, which we will organize according to the number of inversions $(0,1,2, \ldots)$.

(2) There is an oriented edge from $p^{\prime}$ to $p^{\prime \prime}$ if $p^{\prime \prime}$ can be obtained by means of an inverse transposition in $p^{\prime}$, or equivalently (see theorem 7.11) if $Y\left(p^{\prime \prime}\right)$ is an uniparametric perturbation of $Y\left(p^{\prime}\right)$.

We refer to it as the graph of marked perturbations in $\operatorname{Inv}(p, q)$.

Example 7.13 For $p=(6,4,3,2)$ and $q=(4,2,1,0)$ the graph of marked perturbations is represented in figure 1. For example, a possible path is: $(6,4,3,2),(6,4,2,3),(6,2,4,3),(4,2,6,3)$, $(4,2,3,6)$. Notice that the edge $(4,6,3,2),(4,2,3,6)$ increases in 3 units the number of inversions.

Clearly, the graph of marked perturbations has origin $\left(p_{1}, \ldots, p_{n}\right)$ and the graph ends in the compatible permutation having maximal number of inversions (in the above example, $(4,2,3,6)$ ). We can restrict ourselves to the subgraph ending in any prefixed vertex.

Example 7.14 For the above graph, the subgraph ending in $Y(4,2,6,3)$ is represented in figure 1 , by means of discontinuous arrows.

Finally, given an invariant subspace $\mathcal{V} \in \operatorname{Inv}(p, q)$ we consider the marked subspaces such that $\mathcal{V}$ is a perturbation of each of them. It is clear that it is also a subgraph, with origin $\left(p_{1}, \ldots, p_{n}\right)$ and theorem 7.11 shows that its end is the marked type of $\mathcal{V}$. In addition, we point out that if the initial subspace lies in the boundary of the class of the perturbed one, the dimension of its orbit is smaller than the one of the perturbed one. Therefore we conclude:

Corollary 7.15 Given $\mathcal{V} \in \operatorname{Inv}(p, q)$, its marked type is the one whose class has the maximal dimension among the marked subspaces lying in the boundary of its class.

Example 7.16 Let us consider $p=(4,3,1), q=(2,1)$. Then $\operatorname{Inv}(p, q)$ is 3-dimensional and fully parametrised by the miniversal deformation of $Y(4,3,1)$ : 


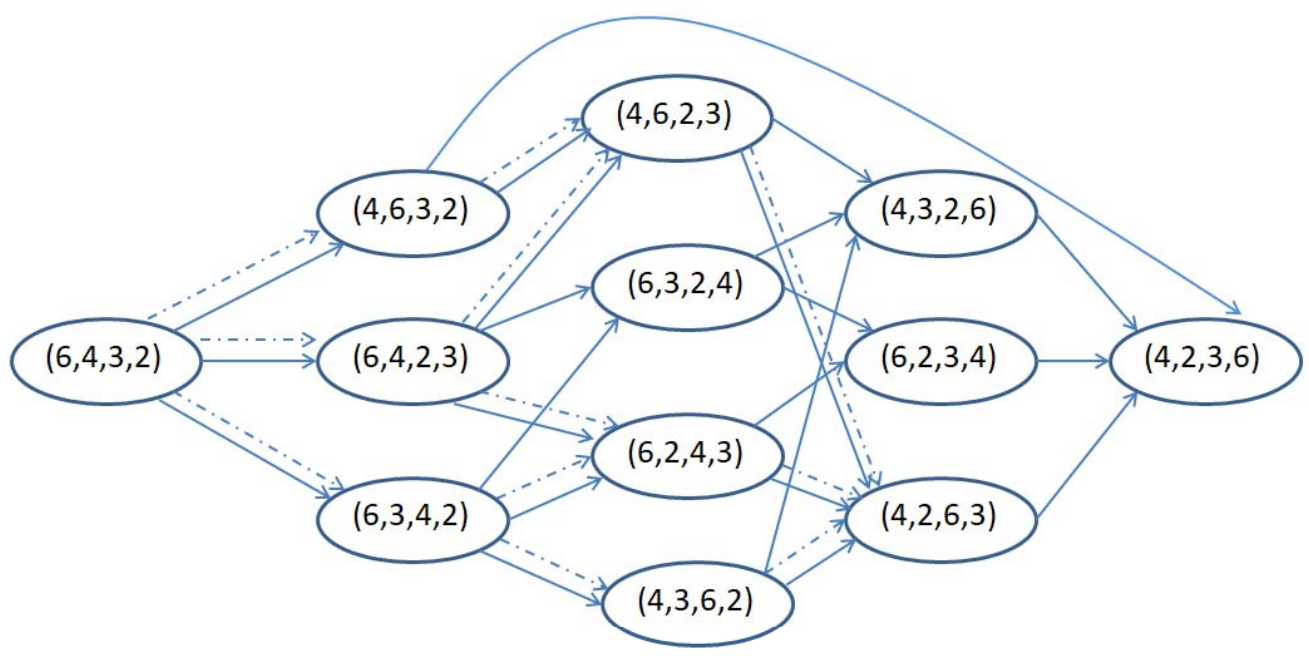

Fig. 1: Graph (with continuous arrows) and subgraph (with discontinuous arrows) corresponding to example 7.13 and example 7.14 , respectively.

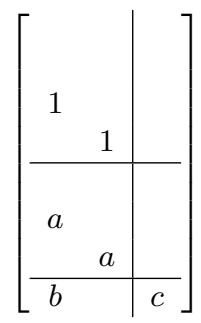

The marked classes are: \begin{tabular}{c|c|c|}
\hline values & classes & dimension \\
$a=b=c=0$ & $Y(4,3,1)$ & 0 \\
$a \neq 0, b=c=0$ & $Y(3,4,1)$ & 1 \\
$c \neq 0, a=b=0$ & $Y(4,1,3)$ & 2 \\
$a c \neq 0$ & $Y(3,1,4)$ & 3 \\
\hline
\end{tabular}

\begin{tabular}{c|c|c|}
\cline { 2 - 3 } The non-marked classes are: & values & classes \\
$a=c=0, b \neq 0$ & $\tilde{Y}_{1}$ \\
$a \neq 0, b \neq 0, c=0$ & $\tilde{Y}_{2}$ \\
$a=0, b \neq 0, c \neq 0$, & $\tilde{Y}_{3}$ \\
\hline
\end{tabular}

Only $Y(4,3,1)$ lies in the boundary of $\tilde{Y}_{1}$. Both $Y(4,3,1)$ and $Y(3,4,1)$ lie in the boundary of $\tilde{Y}_{2}$ : the marked type of $\tilde{Y}_{2}$ is $Y(3,4,1)$. Both $Y(4,3,1)$ and $Y(4,1,3)$ lie in the boundary of $\tilde{Y}_{3}$ : its marked type is $Y(4,1,3)$.

\section{Acknowledgments}


We are very grateful to the referees for their suggestions and to Dr. Antoni Susín for his collaboration.

\section{References}

[1] V.I. Arnold, On Matrices Depending on Parameters, Uspekhi Mat. Nauk., 26 (1971), p. $101-114$.

[2] A. Compta; J. Ferrer, A Geometric Approach to the Carlson Problem, SIAM Journal on Matrix Analysis Appl. Vol. 22, n. 1(2000), pp. 258-275.

[3] A. Compta; J. Ferrer, Matricial Realizations of the Solutions of the Carlson Problem, Linear Algebra Appl., 353 (2002), pp. 197-206.

[4] A.Compta; J. Ferrer, Geometric classification of monogenic subspaces and uniparametric linear control systems, Linear and multilinear algebra, 63 - 9 (2015), p. 1768-1785.

[5] A. Compta; J. Ferrer; M. PeÑA, Dimension of the orbit of marked subspaces, Linear Algebra Appl., 379 (2004), p. 239-248.

[6] A. Compta; J. Ferrer; F. Puerta, Miniversal deformations of marked matrices, Linear Algebra Appl., 361 (2003), p. 181-201.

[7] A. Compta; U. Helmke; M. Peña; X. Puerta, Simultaneous versal deformations of endomorphisms and invariant subspaces, Linear Algebra Appl., 413 (2006), p. 303318

[8] J. Ferrer; F. Puerta, Versal Deformations of Invariant Subspaces, Linear Algebra Appl., 332-334 (2001), p. 569-582.

[9] J. Ferrer; F. Puerta; X. Puerta, Geometric Characterization and Classification of Marked Subspaces, Linear Algebra Appl., 235 (1996), p. 15-34.

[10] I. Gohberg; P. Lancaster; L. Rodman, Invariant Subspaces of Matrices with Applications. Wiley, New York (1986).

[11] I. Kaplansky, Infinite Abelian Groups. Ann Arbor (MI): University of Micihigan Pres; 1954.

[12] C.M. Ringel; M. Schmidmeier, Invariant subspaces of nilpotent operators. I., Journal Reine Angew. Math. Band, 614 (2008), p. 1-52.

[13] M.A. Shayman, On the variety of invariant subspaces of a finite-dimensional linear operator, trans, Amer. Math. Soc., vol. 274 (2) (1982), p. 721-747. 